\title{
Multifractal earth topography
}

\author{
J.-S. Gagnon ${ }^{1}$, S. Lovejoy ${ }^{1,2}$, and D. Schertzer ${ }^{3}$ \\ ${ }^{1}$ Department of Physics, McGill University, Montréal, Canada \\ ${ }^{2}$ Centre GEOTOP UQAM/McGill, Université du Québec à Montréal, Montréal, Canada \\ ${ }^{3}$ CEREVE, École Nationale des Ponts et Chaussées, Marne-la-Vallée, France
}

Received: 23 May 2006 - Revised: 11 September 2006 - Accepted: 1 October 2006 - Published: 16 October 2006

\begin{abstract}
This paper shows how modern ideas of scaling can be used to model topography with various morphologies and also to accurately characterize topography over wide ranges of scales. Our argument is divided in two parts. We first survey the main topographic models and show that they are based on convolutions of basic structures (singularities) with noises. Focusing on models with large numbers of degrees of freedom (fractional Brownian motion ( $\mathrm{fBm}$ ), fractional Levy motion (fLm), multifractal fractionally integrated flux (FIF) model), we show that they are distinguished by the type of underlying noise. In addition, realistic models require anisotropic singularities; we show how to generalize the basic isotropic (self-similar) models to anisotropic ones. Using numerical simulations, we display the subtle interplay between statistics, singularity structure and resulting topographic morphology. We show how the existence of anisotropic singularities with highly variable statistics can lead to unwarranted conclusions about scale breaking.

We then analyze topographic transects from four Digital Elevation Models (DEMs) which collectively span scales from planetary down to $50 \mathrm{~cm}$ (4 orders of magnitude larger than in previous studies) and contain more than $2 \times 10^{8}$ pixels (a hundred times more data than in previous studies). We use power spectra and multiscaling analysis tools to study the global properties of topography. We show that the isotropic scaling for moments of order $\leq 2$ holds to within $\pm 45 \%$ down to scales $\approx 40 \mathrm{~m}$. We also show that the multifractal FIF is easily compatible with the data, while the monofractal $\mathrm{fBm}$ and $\mathrm{fLm}$ are not. We estimate the universal parameters $\left(\alpha, C_{1}\right)$ characterizing the underlying FIF noise to be $(1.79$, $0.12)$, where $\alpha$ is the degree of multifractality $(0 \leq \alpha \leq 2,0$ means monofractal) and $C_{1}$ is the degree of sparseness of the surface $\left(0 \leq C_{1}, 0\right.$ means space filling). In the same way, we investigate the variation of multifractal parameters between
\end{abstract}

Correspondence to: J.-S. Gagnon

(gagnonjs@physics.mcgill.ca) continents, oceans and continental margins. Our analyses show that no significant variation is found for $\left(\alpha, C_{1}\right)$ and that the third parameter $H$, which is a degree of smoothing (higher $H$ means smoother), is variable: our estimates are $H=0.46,0.66,0.77$ for bathymetry, continents and continental margins. An application we developped here is to use ( $\alpha$, $C_{1}$ ) values to correct standard spectra of DEMs for multifractal resolution effects.

\section{Introduction}

\subsection{Models and descriptions}

The Earth's topography is extremely variable over wide ranges of space-time scales. It strongly varies from one location to another and from one scale to another, making it hard to tackle with classical (scale bound) geostatistics. The development of realistic descriptions and models of topography has long been a basic challenge not only to geoscientists, but also to physicists and mathematicians (e.g. Perrin (1913)). An accurate description of topography could be used to put constraints on any first principles geophysical model of topography, shed some light on the internal mechanisms of the Earth and help explain many aspects of surface hydrology. More practically, such a description could also be used as input in various applications involving topography/bathymetry as a boundary condition. Examples include the use of a random bathymetry model as input in a simplified oceanic currents model (Alvarez et al., 2000), Guarnieri (2002) who uses multifractal models in synthetic aperture radar interferograms and Orosei et al. (2003) who use monofractals in models of radar scattering by the Martian surface.

The problem is that descriptions and models are fundamentally linked. On the one hand, an accurate description of topography is needed to place constraints on geophysical models; on the other hand, without a basic model of the

Published by Copernicus GmbH on behalf of the European Geosciences Union and the American Geophysical Union. 
topography, it is not clear what type of description should be sought. Should a characterization of the topography and its morphology necessarily contain a wide range of scales, or is it meaningful to filter out all but a narrow range and focus on characterizing and modeling these while ignoring the others? The conundrum of requiring a model simply in order to analyze and characterize superficially "raw" data is well illustrated by the development of scaling ideas and their applications to topography. Even if we limit ourselves to scaling characterizations and models, we still need more precise ideas about the types of scaling (isotropic, anisotropic, monofractal, multifractal). Indeed, we argue that overly restrictive (isotropic, monofractal) frameworks have lead numerous researchers to throw out the baby with the bathwater, effectively dismissing all wide range scaling approaches as unrealistic.

\subsection{Scaling in topography}

The quantitative use of scaling laws in topography goes back at least to Vening Meinesz (1951), who used the spherical harmonic expansion of the Earth's topography of Prey (1922) to show that the power spectrum $E(k)$ of topography (where $k$ is a wavenumber) roughly follows a power law $k^{-\beta}$ with a spectral exponent $\beta \approx 2$ (the original results are in Vening Meinesz (1951), but the essential points that are quoted here can be found in Heiskanen and Vening Meinesz (1958)). After his pioneering work, Balmino et al. (1973) made similar analyzes on more modern data sets and confirmed Vening Meinesz's results. Bell (1975) followed, combining various data sets (including those of abyssal hills) to produce a composite power spectrum that was scaling over approximately 4 orders of magnitude in scale also with $\beta \approx 2$ (here and below we use the exponent of the angle integrated spectrum; the angle averaged spectrum has exponent $\beta+D-1$, where $D=2$ is the dimension of space). More recent spectral studies of bathymetry over scale ranges from $0.1 \mathrm{~km}$ to $1000 \mathrm{~km}$ can be found in Berkson and Matthews (1983) $(\beta \approx 1.6-1.8)$, Fox and Hayes (1985) $(\beta \approx 2.5)$, Gibert and Courtillot (1987) $(\beta \approx 2.1-2.3)$ and Balmino (1993) $(\beta \approx 2)$. Attempts were even made (Sayles and Thomas, 1978) to generalize this to many natural and artificial surfaces: the resulting spectrum exhibited scaling over 8 orders of magnitude with $\beta \approx 2$ (see however the critique by Berry and Hannay (1978) and Sect. 6.2.2).

If the topography has a power law spectrum, then isolines (such as coastlines) are fractal sets, they have no tangent (Perrin, 1913) and are nonrectifiable (infinite in length) (Steinhaus, 1954). In particular, Richardson (1961) found that the length of various coastlines varies in a power law way with the length of the rulers used to measure them. Mandelbrot (1967), in his famous paper "How long is the coast of Britain", interpreted these scaling exponents in terms of fractal dimensions. Later, with the advent of fractional Brownian motion (fBm) models of terrain (Mandelbrot, 1975; Good- child, 1980), many fractal studies of topography were made as well as the corresponding (gaussian) simulations of topography.

Since then, there have been many indirect estimates of (supposedly unique) fractal dimensions on topographic transects and surfaces using various methods to see if topography respects "fractal" statistics. The indirect methods start by postulating a priori that a unique fractal dimension exists, and then exploit special monofractal relations to deduce the presumed unique fractal dimension from structure functions (variograms), power spectra or other statistical exponents; see for example Burrough (1981); Mark and Aronson (1984) for the variogram method, Gilbert (1989); Huang and Turcotte $(1989,1990)$ for the power spectrum method and Dietler and Zhang (1992) for the "roughening exponent" method. See also Klinkenberg and Goodchild (1992); Xu et al. (1993); Gallant et al. (1994) for reviews and discussions of the results of such monofractal processes.

In contrast to indirect monofractal based inference, direct estimates of fractal dimensions of topography and bathymetry (using box-counting for example) are surprisingly rare (e.g., Barenblatt et al., 1984; Aviles et al., 1987; Okubo and Aki, 1987; Turcotte, 1989). For monofractal fields (such as $\mathrm{fBm}$ ), the box dimension is independent of the threshold used to define the set; Lovejoy and Schertzer (1990) show that for topography this is quite unrealistic. Analyzing the topography of France at $1 \mathrm{~km}$ resolution, they showed that the box dimension systematically decreases from 2 (the maximum possible) to 0 (the minimum) as the altitude is increased. This shows that monofractals are at best an approximation of topography near the mean.

As argued in Lovejoy and Schertzer (1990); Lavallée et al. (1993), it is more appropriate to treat topography as a scale invariant field, generally requiring multifractal measures and exponent functions (rather than a unique scaling exponent, such as the fractal dimension). An infinity of fractal dimensions (one for each threshold or equivalently one for each statistical moment) are then needed to completely characterize the scaling. A few multifractal studies of topography that show that it is multiscaling in various regions of the world and over various ranges in scale can be found in Lovejoy and Schertzer (1990); Lavallée et al. (1993); Weissel et al. (1994); Lovejoy et al. (1995); Pecknold et al. (1997); Tchiguirinskaia et al. (2000); Gagnon et al. (2003). A similar mono vs multifractal issue also arises in the study of fractures and other artificial surfaces (e.g., Morel et al., 2000); while the monofractal model is quite popular, isolated results (Bouchaud et al., 1993; Schmittbuhl et al., 1995) point to multifractality.

There is also much indirect evidence for the scaling of the topography. For example the albedoes and surface emissions at different wavelengths are nonlinearly coupled with the topography over wide ranges of scales. Since scale invariance is a symmetry principle, if there is a break in the scaling of the topography it should be observed in the lat- 
ter and vice versa. The findings of Harvey et al. (2002) and Gaonac'h et al. (2003) that the remotely sensed radiation fields from volcanoes are multifractal therefore suggest the multifractality of the corresponding topographies. Similarly, the scaling of surface magnetic susceptibility (Pilkington and Todoeschuck, 1995), rock density (Leary, 1997; Lovejoy et al., 2005), and the multiscaling of geomagnetism (Lovejoy et al., 2001a; Pecknold et al., 2001) and rock sonic velocities (Marsan and Bean, 1999) are all relevant.

Other indirect evidence in favor of scaling and multiscaling of the topography comes from hydrology, as can be seen from the abundant literature on the scaling of river basin geomorphology (see in particular Rodriguez-Iturbe and Rinaldo (1997) and the references therein). This includes the classical scaling of river slopes, lengths, discharges and widths with respect to the area of the drainage basins, but also to the scaling (Hurst, 1951; Mandelbrot and van Ness, 1968) and multiscaling (Tessier et al., 1996; Pandey et al., 1998) of the temporal variation of river discharges. While the river basin geomorphology relations suggest the scale invariance of many orographic/erosion processes, the scale invariance of the discharges suggests the scale invariance of topography/runoff/infiltration processes. Indeed, the ubiquity of scaling relations in surface hydrology would be difficult to comprehend without wide range scale invariance of the topography.

\subsection{Models of topography}

The topography of the Earth is very complex and its morphology results from diverse processes, notably tectonic forces (faulting, folding, flexure) and erosion, under the influence of gravity and other factors (Turcotte, 1992; Lambeck, 1988, and references therein). Although "equations", i.e. nonlinear partial differential equations, describing the evolution of topography are not known, some models exist to explain certain of its features. Such physical models can be divided in two main categories: those with few degrees of freedom and those with many. The former are generally deterministic and model narrow ranges of scale, whereas the latter are generally stochastic and cover wider ranges of scale. To date, deterministic geodynamic equations, introducing physical characteristic scales at the beginning, have attempted to model the topography over only a fairly narrow range of scales. At best, they predict the general trend of certain features of topography but do not predict its rugged aspect nor its fine structure. For example, the large swell around seamount chains can be explained by thermal expansion of the lithosphere caused by a heat source in the mantle (hotspot, plume; see for example Lambeck (1988)). Another well known example is the bathymetry of the sea floor associated with mid-ocean ridges. The ridges are sources of hot material coming from the mantle that create new oceanic crust. The material injected at the ridge crest cools off, contracts and moves away as part of the plate, creating the char- acteristic topography of the sea floor. In the framework of plate tectonics, Turcotte and Oxburgh (1967) (see Parsons and Sclater (1977) for a review) have reproduced the characteristic decrease of the altitude from the ridge using the equation of heat transport with appropriate boundary conditions, giving $\Delta h \propto \Delta x^{1 / 2}$ (see Table 1). As can be seen from Parsons and Sclater (1977), the general approach is to start with a set of linear or nonlinear partial differential equations and simplify them (by making various assumptions and approximations) so that they can be solved. These deterministic models are generally too linear to explain the variability of topography (Mareschal, 1989), a consequence of the homogeneity hypotheses that reduce the problem to a small number of degrees of freedom. For example, in the thermal boundary layer model of Turcotte and Oxburgh (1967), the mantle is considered to be "smooth" below the length scale of a convective cell.

To take into account the high number of degrees of freedom and the variability over a wide range of scales, it is natural to use stochastic approaches which are typically based on infinite dimensional probability spaces. For example, Bell (1975) uses hills with random sizes that are uniformly distributed over the bottom of the ocean to model bathymetry (excluding mid-ocean ridges). Because the geodynamic equations considered here are difficult to solve without approximation, it is fruitful to consider one of the symmetries of the problem (i.e. scale invariance), which empirically approximates the topography over wide ranges (see Sect. 1.2).

There are two main approaches to the problem depending on if we are interested in modeling specific processes or rather the overall outcome of all the topographic processes. The first approach is mainly used to represent topography within river basins and aims at modeling the effect of specific landsculpting processes (such as fluvial erosion, sediment deposition, diffusion, etc) on topography and drainage networks. For example, in this context Chase (1992) presents a model that can produce topography with scaling properties consistent with observations. Another model that uses scaling as a basic principle in addition to stochasticity is the phenomenological Kardar Parisi Zhang (KPZ) equation (Kardar et al., 1986). It was originally introduced to study growing and eroding surfaces, but it is also used to model topography (Sornette and Zhang, 1993) (see also Dodds and Rothman (2000) for a pedagogical introduction). Other tools used to study the causes of topographic scaling includes selforganized criticality (Rinaldo et al., 1993), minimization of energy functionals (Rinaldo et al., 1992, 1996; Sinclair and Ball, 1996; Banavar et al., 2001) and renormalization properties of fluvial erosion equations (Veneziano and Niemann, 2000a,b). An extensive review of the use of diffusion-like equations to model topography within river basins can be found in Rodriguez-Iturbe and Rinaldo (1997).

The second approach is more aimed at reproducing accurately the statistics of topography, usually over wide ranges of scales. One such popular stochastic approach based on 
Table 1. An intercomparison between various models of the topography showing the essential similarities and differences. For additional information about notation and definitions, see Table 2 and Sect. 3 . Here $D=2$ for horizontal planes and the dimension $D_{F}$ is the fractal dimension of lines of constant altitude in the horizontal. The deterministic mid-ocean ridge model is represented here by a fault in unit direction vector $\mathbf{r}$ through the point $\mathbf{x}_{0}$. Here the variables are nondimensionalized and the height of the fault is normalized to one. Note that $\delta$ is a Dirac delta function. The model of Turcotte and Oxburgh (1967) uses $H=1 / 2$. The monofractal fBm model is characterized by a fractional integration of order $H^{\prime}$ of a Gaussian white noise with variance $\sigma^{2}$. It can also be produced by simply summing over large numbers or random Gaussian distributed faults (see Fig. 1). Here $H^{\prime}=H+D / 2$, where the extra $D / 2$ in the exponent takes into account the scaling of the noise ( $D$ is the dimension of space). The value $H=1 / 2$ is compatible with the commonly cited value $D_{F}=1.5$ for the dimension of topographic level sets. Note that $\stackrel{d}{=}$ means equality in probability distributions. The monofractal fLm model is a generalization of $\mathrm{fBm}$ obtained by replacing the Gaussian white noise with an independent Levy noise of index $\alpha<2$. It has diverging moments for $q \geq \alpha$. Here $H^{\prime}=H+\alpha / 2$. Finally, the multifractal FIF model is a generalization of fBm and fLm. Here the multifractal noise $\phi_{\lambda}$ is the result of a continuous in scale multiplicative cascade. Mathematically, it is given by $\phi_{\lambda}(\mathbf{x})=e^{\Gamma_{\lambda}(\mathbf{x})}$, where the generator $\Gamma_{\lambda}(\mathbf{x}) \propto \int_{1}^{\lambda} d x^{\prime} \frac{\phi_{\alpha}^{\prime}\left(\mathbf{x}^{\prime}\right)}{\left|\mathbf{x}-\mathbf{x}^{\prime}\right|^{D-H^{\prime}}}$ is an fLm process with $H^{\prime}=D(1-1 / \alpha)$ and a maximally skewed Levy noise $\phi_{\alpha}^{\prime}$. The resulting $\phi_{\lambda}$ is multiplicative because it is an exponentiation of the additive process $\Gamma_{\lambda}$.

\begin{tabular}{|c|c|c|c|}
\hline Model & $\begin{array}{l}\text { Altitude } \\
\text { (and noise statistics) }\end{array}$ & $\begin{array}{l}\text { Altitude increments } \\
\text { (and statistics) }\end{array}$ & $\begin{array}{l}\text { Codimension (c) } \\
\text { of level sets }\end{array}$ \\
\hline $\begin{array}{l}\text { Mid-ocean ridge } \\
\text { (deterministic) }\end{array}$ & $\begin{array}{l}h(\mathbf{x})=1-\int d \mathbf{x}^{\prime} \frac{\delta\left(\mathbf{x}^{\prime}-\mathbf{x}_{0}\right)}{\left|\mathbf{r} \cdot\left(\mathbf{x}-\mathbf{x}^{\prime}\right)\right|^{-H}} \\
\text { (No noise statistics) }\end{array}$ & $\begin{array}{l}\Delta h \propto|\Delta \mathbf{x}|^{H} \\
\text { (No altitude statistics) }\end{array}$ & $\begin{array}{l}c=D-D_{F} \\
D_{F}=1\end{array}$ \\
\hline $\begin{array}{l}\text { Monofractal fBm } \\
\text { (stochastic) }\end{array}$ & $\begin{array}{l}h(\mathbf{x})=\int d \mathbf{x}^{\prime} \frac{\phi_{2}\left(\mathbf{x}^{\prime}\right)}{\left|\mathbf{x}-\mathbf{x}^{\prime}\right|^{D-H^{\prime}}} \\
\phi_{2}(\mathbf{x})=\text { Gaussian white noise } \\
H^{\prime}=H+D / 2\end{array}$ & $\begin{array}{l}\Delta h \stackrel{d}{=} \phi_{2}|\Delta \mathbf{x}|^{H} \\
\left\langle|\Delta h|^{q}\right\rangle \propto|\Delta \mathbf{x}|^{\xi(q)} \\
\xi(q)=q H\end{array}$ & $\begin{array}{l}c=H \\
D_{F}=D-c\end{array}$ \\
\hline $\begin{array}{l}\text { Monofractal fLm } \\
\text { (stochastic) }\end{array}$ & $\begin{array}{l}h(\mathbf{x})=\int d \mathbf{x}^{\prime} \frac{\phi_{\alpha}\left(\mathbf{x}^{\prime}\right)}{\left|\mathbf{x}-\mathbf{x}^{\prime}\right|^{D-H^{\prime}}} \\
\phi_{\alpha}(\mathbf{x})=\text { Levy noise }(0 \leq \alpha \leq 2) \\
H^{\prime}=H+D / \alpha\end{array}$ & $\begin{array}{l}\Delta h \stackrel{d}{=} \phi_{\alpha}|\Delta \mathbf{x}|^{H} \\
\left\langle|\Delta h|^{q}\right\rangle \propto|\Delta \mathbf{x}|^{\xi(q)} \\
\xi(q)=\left\{\begin{array}{cc}q H & \text { for } q<\alpha \\
\infty & \text { for } q \geq \alpha\end{array}\right.\end{array}$ & $\begin{array}{l}c=H \\
D_{F}=D-c\end{array}$ \\
\hline $\begin{array}{l}\text { Multifractal FIF } \\
\text { (stochastic) }\end{array}$ & $\begin{array}{l}h_{\lambda}(\mathbf{x})=\int d \mathbf{x}^{\prime} \frac{\phi_{\lambda}\left(\mathbf{x}^{\prime}\right)}{\left|\mathbf{x}-\mathbf{x}^{\prime}\right|^{D-H}} \\
\left\langle\phi_{\lambda}^{q}\right\rangle=\lambda K(q)\end{array}$ & $\begin{array}{l}\Delta h=\phi_{\lambda}|\Delta \mathbf{x}|^{H} \\
\left\langle|\Delta h|^{q}\right\rangle \propto|\Delta \mathbf{x}|^{\xi(q)} \\
\xi(q)=q H-K(q)\end{array}$ & $\begin{array}{l}c(\gamma)=\max _{q}\{q H-K(q)\} \\
D_{F}(\gamma)=D-c(\gamma)\end{array}$ \\
\hline
\end{tabular}

scale invariance is the fractional Brownian motion ( $\mathrm{fBm}$ ) model of topography (Mandelbrot, 1975). In this model, topography is obtained by fractionally integrating a Gaussian white noise (see Table 1). In comparison, the fractionally integrated flux (FIF) model (Schertzer and Lovejoy, 1987) can be viewed as a multifractal generalization of $\mathrm{fBm}$ : it is obtained by replacing the Gaussian white noise $\phi$ (with no particular relation between different scales) with a scaling multifractal noise $\phi_{\lambda}$ (i.e. with long range statistical dependencies). This multifractal noise is the result of a cascade (see Sect. 3.1), which is a scale invariant random multiplicative process (Table 1). All of the models must be strongly anisotropic to be realistic.

\subsection{Objectives of the present study}

For more than half a century, scaling has been a feature of many topographic models. With the advent of fractals in the 70's, the significance of scaling became even more widely appreciated, especially due to the impressive fractal simulations in Mandelbrot (1975, 1983). Unfortunately, the initial enthusiasm inspired by the images and the theoretical simplicity of a model with a single basic exponent (the supposedly unique fractal dimension) had by the end of the 90's been largely dissipated. In place of the larger picture, focus had turned to purely technical issues: over which scale range?, which fractal dimension?, what link with location or type of terrain?, what analysis methods?, etc. The problem was that the early scaling notions were simply inadequate. Two aspects in particular were totally unrealistic: the first is monofractality (uniqueness of $D_{F}$ ) and the second is the restriction to isotropy ("self-similarity"). This last point is particularly important, because topography possess a variety of morphologies/textures (e.g. mountain ranges) that are highly anisotropic. 
Today, the scale invariance symmetry principle - having been identified with unrealistic and restrictive special cases (i.e. isotropic monofractals) - is typically disregarded or else limited to small ranges of scale. Unsurprisingly, it is considered to be uninteresting and unphysical. However, the history of symmetry principles shows that their power should not be underestimated. The example of the symmetry principle of energy conservation is instructive. At first confined to mechanical energy, it was generalized at the end of the 18th century to include heat energy. As knowledge progressed, scientists were repeatedly faced with the choice of either abandoning it or generalizing it. By the mid 19th century it included chemical and electrical energy and - even though as a general principle, it was initially criticized as being overly speculative and philosophical - it was proposed as a universally valid physical law. In the 20th century, it continued to be generalized to include mass-energy.

In this paper, we argue that two key advances make scaling applicable to topography: the generalization from mono to multifractals and the generalization from isotropic to anisotropic scaling. Our argument is divided in two parts. In the first part of the paper, we survey and contrast various topographic models (both deterministic and stochastic), emphasizing their similar mathematical structures. We also explain, with the help of simulations, how one can obtain a wide diversity of morphologies/textures by exploiting scaling and letting the geodynamics determine the notion of scale; this is done in the framework of Generalized Scale Invariance (Schertzer and Lovejoy, 1985). The simulations allow us to begin the exploration of the subtle interplay between singularities, structures, statistics and overall morphologies. We also clarify some misconceptions about multifractals and multifractal variability with the help of simulations and pedagogical examples. Among other things, we show how superficially conflicting monofractal results can be understood as a result of limited statistics combined with multifractal variability.

In a previous paper (Gagnon et al., 2003), we concentrated on the mono vs. multi fractal issue in rough surface physics using topography as an example. We clearly demonstrated that on purely statistical grounds, monofractals are not sufficient to describe topography and that multifractals are needed. We elaborate on this in the second part of the paper. In addition, the use of the large topographic data sets that have become available in the last few years allows the investigation of two issues which go beyond the mono versus multifractal debate.

The first issue concerns the nature of the differences between continents and oceans. Continents and oceans do not have the same geological history, so that their topographies are probably the result of qualitatively different (but still possibly scaling) processes. For example, the erosion on continents is due to water, wind and glaciers, whereas in the oceans it has probably been due to marine currents (little erosion, mostly sediment deposition).
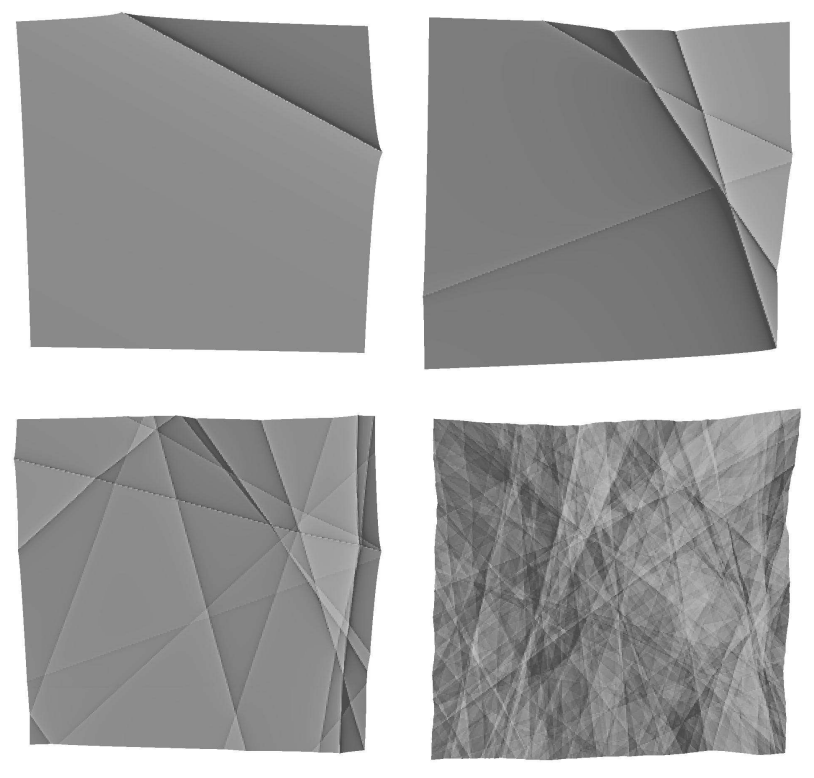

Fig. 1. Numerical simulation of Mandelbrot (1975)'s random fault implementation of fBm. The faults are power-law shaped (see Table 1, first and second rows) and their location and orientation are randomly chosen. The simulation is done with Gaussian statistics and $H=1 / 2$, showing development with 1, 4, 16 and 256 faults (left to right, top to bottom). The Turcotte and Oxburgh (1967) model is in the upper left corner (one fault case).

The second issue concerns the multiscaling and the "global" properties of topography. More precisely, the large range of scales of this study allows us to quantify the extent of the range of topographic multiscaling and to characterize its isotropic (angle averaged) statistics with just three fundamental exponents $\alpha, C_{1}$ and $H$ (see Sects. 3.2 and 3.3 for their definitions and meanings).

The rest of the paper is organized as follows. Section 2 discusses the key issues of singularities, statistics and morphology with many pedagogical examples, the aim of which is to convince the reader that an anisotropic scaling framework is potentially capable of explaining/modelling the topography. Section 3 introduces some notions of multifractal theory. Sections 4 and 5 present, respectively, the data sets and the analysis techniques (power spectrum and trace moments) used in the present study. Section 6 is the core of the paper and presents the results of our analyses. We finally summarize our results and conclude in Sect. 7.

\section{Singularities and morphology}

\subsection{A survey of scaling models of topography}

When over a range of scales, the topography has no characteristic scale, it is natural to model it using combinations 
of scale invariant basis functions, i.e. mathematical singularities. Perhaps the most famous such singular model is the Turcotte-Oxburg model for the variation of altitude as a function of distance from mid-ocean ridges (Turcotte and Oxburgh, 1967). Mathematically the form is indicated in Table 1 (first row) and a simulation is displayed in Fig. 1 (upper left hand corner). Mandelbrot (1975) generalized this model by making singular faults the basic shapes and then summing large numbers of faults with random centers and orientations with Gaussian distributed amplitudes. The result is a Gaussian process with long range (power law) correlations; Fig. 1 shows the first few construction steps. Due to the central limit theorem (the gaussian special case), a process with the same statistical properties can be produced by using singularities of a quite different shape. Table 1 (second row) indicates a model with point rather than line singularities; in this form the mathematics is more convenient for comparison with the other singular topography models summarized in Table 1. In this case, in the limit of many faults, because all of the singularities have nearly the same amplitude (Gaussian variables are rarely more than a few standard deviations from the mean), the basic singularity shape is not important: we end up with a rough texture but without any more interesting morphologies (see Fig. 2, second row).

Notice that in Table 1, all the stochastic models are obtained by convolutions with singularities. Such convolutions are "fractional integrations" of order $H^{\prime}$ (if $H^{\prime}<0$, it is a fractional differentiation). The lesson from $\mathrm{fBm}$ is that if we are to represent real topography by such singular models, then the statistics of the singularities must be more extreme than Gaussians so that the basic singularity shape may remain important in the limit of a large number of singularities. One way to make some of the singularities always stand out is to use the fractional Levy motion model (fLm) obtained by replacing the Gaussian noise by a Levy noise of index $\alpha$. The Levy random variables can be regarded as a generalization of the Gaussian variables to the case where the variance (second moment) is infinite; they have long probability tails such that the statistical moments $q$ of order $q \geq \alpha$ diverge. Due to the (generalized) central limit theorem, sums of independent (possibly weighted) Levy variables are still Levy variables. Figure 2 (third row) shows simulations of fLm. As one can see, several strong mountain peaks stand out; in fact, the strong peaks are too strong. Although far from Gaussian, real topography empirically seems to have finite variance (i.e. the probability density tail falls off faster than $x^{-3}$ ), so fLm cannot be a good model. Before moving on to the statistically and visually more realistic multifractal model, let's consider the singularity shape in more detail. The shape of line (faultlike) and point singularities depends on powers of distances from either a line or a point; in order to generalize this it turns out to be sufficient to replace the standard Euclidean distances by scale functions. Let us therefore digress a moment to discuss scale functions.

\subsection{Scale functions and anisotropic singularities}

In order to change the shape of the singularities while conserving the basic statistical properties of the process, it turns out to be sufficient to make the replacement everywhere in Table 1:

$\left|\mathbf{x}-\mathbf{x}^{\prime}\right| \rightarrow\left\|\mathbf{x}-\mathbf{x}^{\prime}\right\| \quad ; \quad D \rightarrow D_{\mathrm{el}}$

i.e. to replace the usual distance by a new "scale function" and usual dimension of space $D$ by an "elliptical dimension" $D_{\text {el }}$. These new quantities satisfy the following basic scaling equation:

$\left\|T_{\lambda} \mathbf{x}\right\|=\lambda^{-1}\|\mathbf{x}\| ; \quad T_{\lambda}=\lambda^{-G} ; \quad D_{\mathrm{el}}=\operatorname{Trace}(G)$

where $T_{\lambda}$ is a scale changing operator which reduces the scale of a vector by a factor $\lambda$. In order for the scale function to be scaling (i.e. have no characteristic scale), it must satisfy group properties, hence it must admit a generator $G$ as indicated. In the simplest "linear" case, $G$ is a matrix. Once all the unit vectors $\mathbf{x}_{1}$ are specified, the scale equation 2 uniquely specifies the scale of all the other vectors; all the nonunit vectors (i.e. $\left\|\mathbf{x}_{\lambda}\right\|=\lambda$ ) are then generated by the action of $T_{\lambda}$, i.e. $\mathbf{x}_{\lambda}=T_{\lambda} \mathbf{x}_{1}$ (see Schertzer and Lovejoy (1985) for technical details on this Generalized Scale Invariance, GSI). The set of all vectors with scale $\|\mathbf{x}\| \leq \lambda$ is called a "ball", denoted $B_{\lambda}$; for physical scale functions $B_{\lambda}$ must be decreasing (i.e. $B_{\lambda^{\prime}} \subset B_{\lambda}$ for $\left.\lambda^{\prime}<\lambda\right)$. We can see that if the replacements in Eq. (1) are made in the denominators of the models in Table 1, with scale functions satisfying the scale Eq. (2) (in fact they then define the notion of scale), then the convolutions will have power law dependencies under "zooming", i.e. the models will be scaling as long as the noises are also scaling (hence the special choices of Gaussian or Levy noises, or in the multifractal case, of multifractal noises).

To understand the relation between usual distances and generalized scales and see how to find $\|\mathbf{x}\|$ in practice, it is instructive to do an example. To find $\|\mathbf{x}\|$, we need to solve the fundamental Eq. (2). Consider a (real) 2-D $G$ matrix which in a diagonal frame is given by $G=\operatorname{diag}\left(H_{x}, H_{y}\right)$ (note that what follows can be generalized to complex eigenvalues or nondiagonalizable matrices). The idea is to use a nonlinear coordinate transformation to convert the initial problem, i.e. Eq. (2) with $T_{\lambda}=\lambda^{-G}$, into an equivalent problem where $G$ is the identity matrix:

$\left\|\lambda^{-I} \mathbf{x}^{\prime}\right\|=\lambda^{-1}\left\|\mathbf{x}^{\prime}\right\|$

where $\mathbf{x}^{\prime}=\left(x^{\prime}, y^{\prime}\right)$ are the coordinates in the nonlinearly transformed space. It is easy to show that the nonlinear transformation $x^{\prime}=\operatorname{sgn}(x)|x|^{1 / H_{x}}, y^{\prime}=\operatorname{sgn}(y)|y|^{1 / H_{y}}$ does the job for the real diagonal matrix considered above. Equation (3) is now easy to solve: it represents a pure isotropic scale transformation, hence the solution is of the form

$\left\|\mathbf{x}^{\prime}\right\|=\left(\Theta\left(\theta^{\prime}\right)\right)^{-1} r^{\prime}$ 

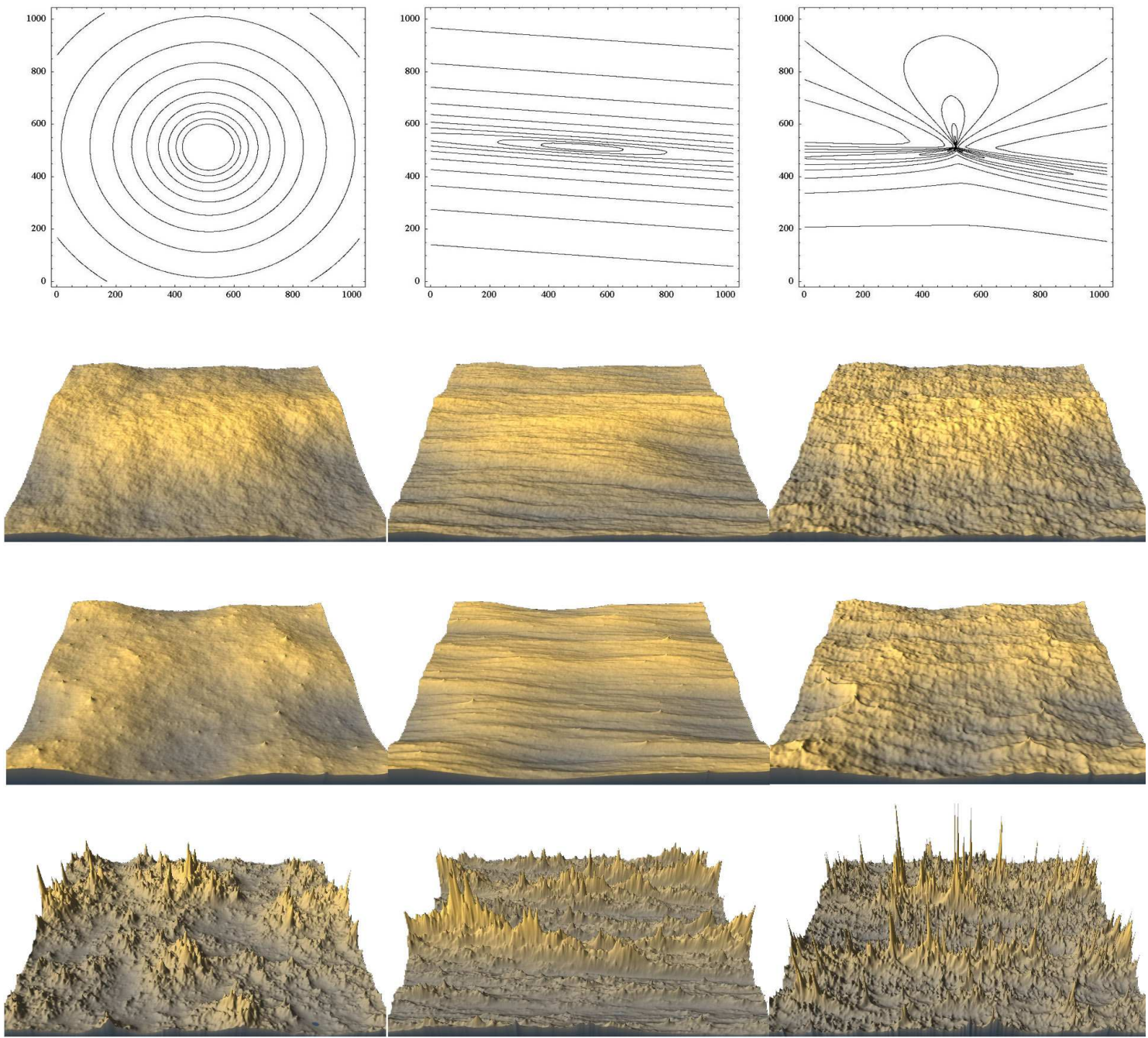

Fig. 2. Comparison of isotropic versus anisotropic (with symmetric scale functions) simulations for three different scaling models. Top row shows scale functions. From left to right, we change the anisotropy: the left column is self-similar (isotropic) while the middle and right columns are anisotropic and symmetric with respect to $G=\left(\begin{array}{cc}0.8 & -0.05 \\ 0.05 & 1.2\end{array}\right)$. The middle column has unit ball circular at 1 pixel, while for the right one it has the form $\Theta\left(\theta^{\prime}\right)=1+0.65 \cos \left(\theta^{\prime}\right)$ (in polar coordinates in the nonlinearly transformed space, see Eq. (4)). Second, third and fourth rows show the corresponding fBm (with $H=0.7)$, fLm $(\alpha=1.8, H=0.7)$ and multifractal $\left(\alpha=1.8, C_{1}=0.12, H=0.7\right)$ simulations. We note that in the case of $\mathrm{fBm}$, one mainly perceives textures, there are no very extreme mountains or other morphologies evident. One can see that the fLm is too extreme, the shape of the singularity (particularly visible in the far right) is quite visible in the highest mountain shapes. The multifractal simulations are more realistic in that there is a more subtle hierarchy of mountains. When the contour lines of the scale functions are close, we change the scale $\|\mathbf{x}\|=\lambda$ rapidly over short (Euclidean) distances. For a given order of singularity $\gamma, \lambda \gamma$ will therefore be larger. This explains the strong variability depending on direction (middle bottom row) and on shape of unit ball (right bottom row). Indeed, spectral exponents will be different along the different eigenvectors of $G$.

where $r^{\prime}=\left(x^{\prime 2}+y^{\prime 2}\right)^{1 / 2}$ is a radial vector in the nonlinearly transformed space and $\Theta\left(\theta^{\prime}\right)$ is an arbitrary function of the polar angle $\theta^{\prime}$ in the nonlinearly transformed space (i.e. $\tan \theta^{\prime}=y^{\prime} / x^{\prime}$ ). The nonlinear coordinate transform is then used on Eq. (4) to obtain the scale function in the original $\mathbf{x}$ space (as opposed to the nonlinearly transformed $\mathbf{x}^{\prime}$ space). For the example considered, the scale function is
$\|\mathbf{x}\|=\left(\Theta\left(\theta^{\prime}\right)\right)^{-1}\left(x^{2 / H_{x}}+y^{2 / H_{y}}\right)^{1 / 2}$.

When scale functions are used to define the basic singularities, the shapes can be extremely varied, hence demonstrating the possibility of modeling topographic morphology in this way. First consider $G=I$, where $I$ is the identity matrix: the resulting topography models will be "self-similar" in the sense that their statistics will vary in power law ways under 

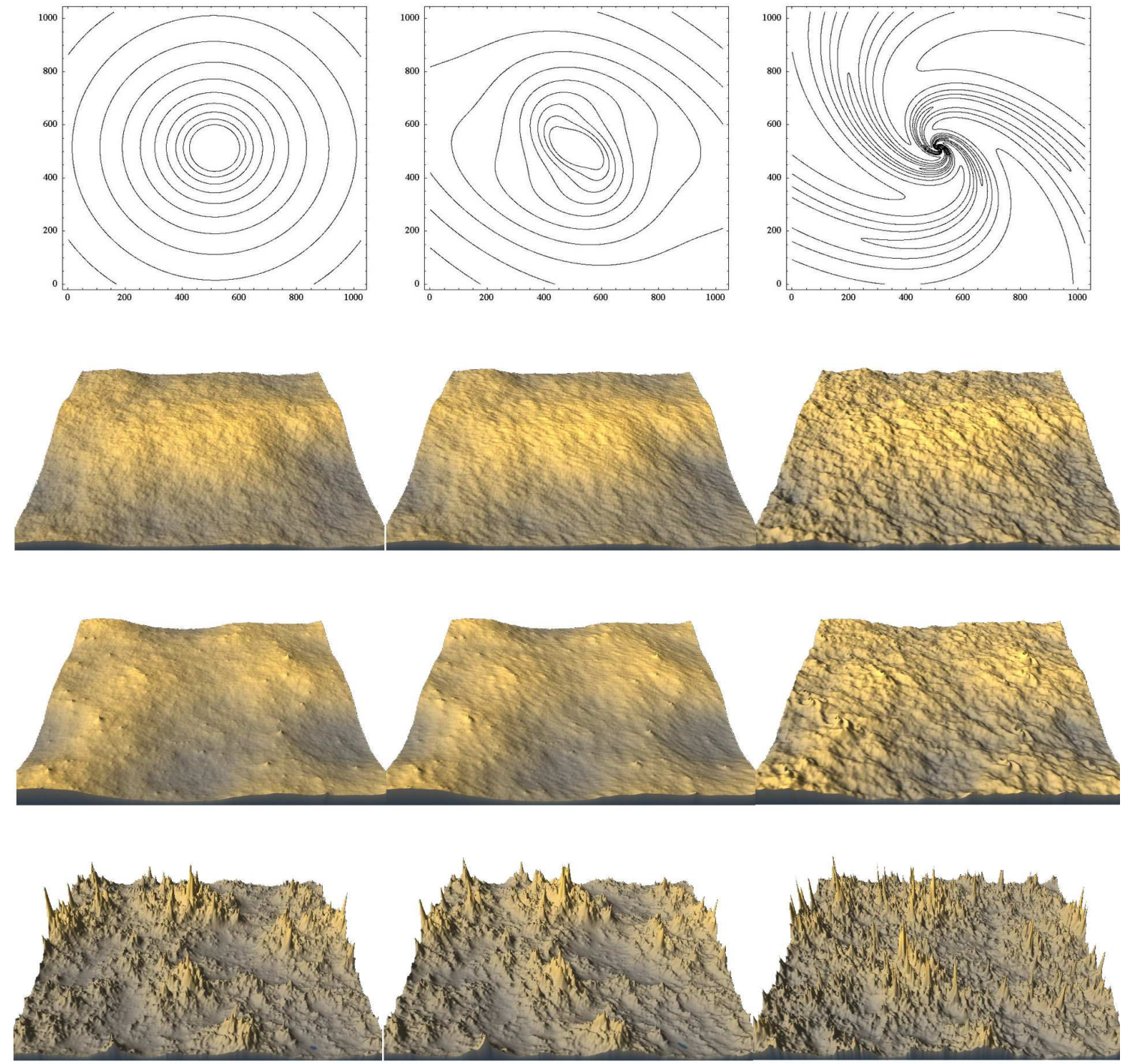

Fig. 3. Comparison of isotropic versus anisotropic (with "spiral" scale functions) simulations for three different scaling models. Top row shows isotropic (left) and spiral (middle, right) scale functions. Spiral scale functions are obtained when $G$ has complex eigenvalues; here $G=\left(\begin{array}{cc}0.5 & -1.5 \\ 1.5 & 1.5\end{array}\right)$. Second, third and fourth rows show the corresponding fBm (with $\left.H=0.7\right)$, fLm $(\alpha=1.8, H=0.7)$ and multifractal $(\alpha=1.8$, $C_{1}=0.12, H=0.7$ ) simulations. Note how the use of spiral singularities does not affect the fBm simulations much (compare with Fig. 2). On the other hand, spiral singularities lead to too strong singularities for the fLm, but subtle variations of mountains and plains for the multifractal.

isotropic "zooming" (blow-ups) (see the left hand column in Fig. 2). When the unit ball is a circle (or more generally a $D$ dimensional sphere), then we obtain $\|\mathbf{x}\|=|\mathbf{x}|$. However when the unit ball is not circular (spherical), then there will still be preferred directions. These preferred directions will be the same at all scales, the anisotropy is "trivial". Things become more interesting as soon as $G$ is no longer the identity. If $G$ is a diagonal matrix, then the singularities $\|\mathbf{x}\|^{-\gamma}$ (where $\gamma$ is the order of singularity, see Sect. 3.1) are quite different in different directions and the resulting fractals/multifractals are "self-affine". The case where $G$ is nondiagonal and the eigenvalues are real is a generalization in which the main stretching/shrinking occurs along fixed nonorthogonal eigendirections; Fig. 2 (middle column) show the resulting differential stratification. When the eigenvalues are complex, then the eigenvectors rotate continuously as functions of scale, giving rise to spiral type singularities, see Fig. 3. Finally, outside our present scope but presumably important for realistic topography modelling, we can consider $G$ as a nonlinear operator (rather than a matrix). In this 
case, the anisotropy depends not only on scale but also on the location. This allows for spatially varying morphologies. In this case, the linear GSI discussed above is simply a locally valid approximation.

\subsection{Spurious breaks in the scaling}

In spite of the systematic finding of scaling or near scaling statistics, many geophysicists reject all wide range scaling, often because of their conviction that geomorphologic processes are scale dependent: they consider a priori that the scaling is broken. For example, Herzfeld et al. (1995); Herzfeld and Overbeck (1999) have attempted to demonstrate broken scaling by estimating power spectra and variograms on a few bathymetry transects which they showed to have poor scaling. Rather than giving a purely theoretical explanation as to why their results are not surprising and how they could be compatible with the scaling hypothesis, let us consider a simulation of their transect (see Fig. 4). The figure compares the energy spectra of two individual transects as well as the ensemble average over all the transects. One of the transects passes through "Mt. Multi" (the highest peak in the range), another through a randomly chosen transect not far away. One can see that the Mt. Multi scaling is pretty poor; a naive analysis would indicate two ranges with a break at about 10 pixels with high frequency exponent $\beta \approx 2.5$, low frequency $\beta \approx 1.5$. Clearly this significant break has nothing to do with the scaling of the process (which is perfect except for finite element effects affecting the highest factor of two or so in resolution). In comparison, the randomly chosen transect has better scaling, but with $\beta \approx 2$. On the other hand, the isotropic (i.e. angle averaged) spectrum averaged over an infinite ensemble of realizations has $\beta \approx 2.17$. Even the average over the transects shows signs of a spurious break at around 16 pixels (the scale where the north-south and east west fluctuations are roughly equal in magnitude, the "sphero-scale"); this explains why the theoretical line does not pass perfectly through the curve corresponding to the average of the transects. Obviously, had we chosen a different random seed for the simulation, the results for the individual transects would have been different (even the average over the transects would have been a bit different), see the example in the next section.

Conclusions about broken scaling in Fig. 4 are therefore unwarranted. The most important reason to explain apparent scaling breaks is that scale invariance is a statistical symmetry, i.e. defined on an infinite ensemble (see Sect. 3.2). This means that scaling is almost surely broken on every single realization, hence it is important to have a large data base (i.e. large range of scales, many realizations) to average fluctuations and approximate the theoretically predicted ensemble scaling. In fact, due to the singularities of all orders (see Sects. 2.1 and 3.1) the realization to realization variability of multifractals is much greater than that of classical stochastic processes; for example, rare (extreme) singularities are
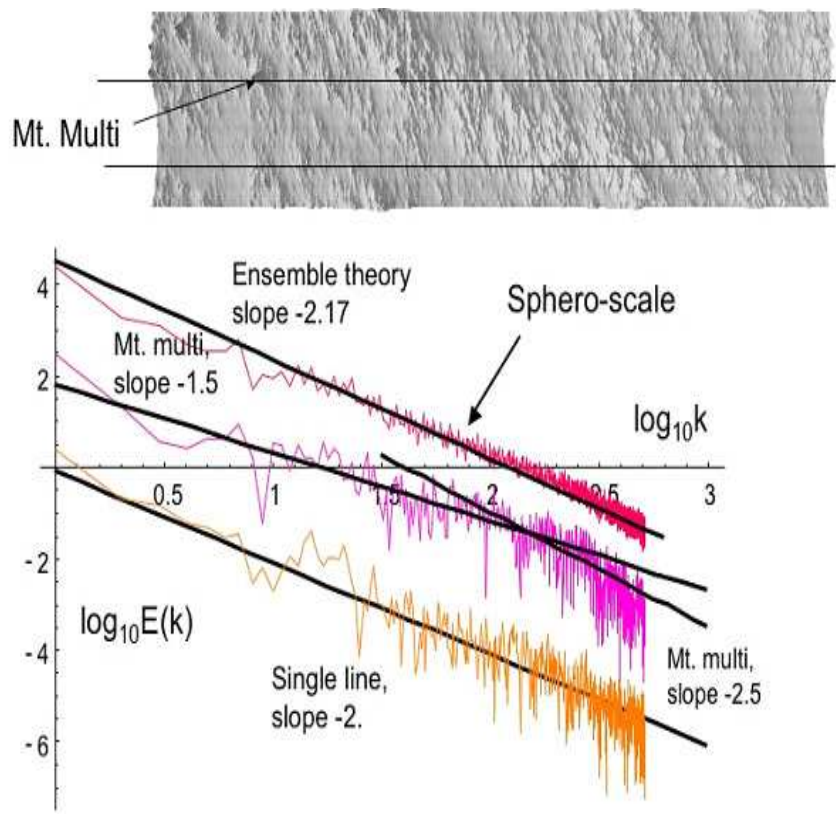

Fig. 4. Figure showing a bathymetry simulation (with $\alpha=1.9$, $C_{1}=0.12$ and $H=0.7$ ). The energy spectra of the transect passing through "Mt. multi" (the highest peak in the simulation) and through another (randomly chosen) transect are shown as well as the ensemble over all the transects. We can see that the scaling in the transect containing the extreme event "Mt. Multi" is clearly broken, even though the ensemble scaling is very good. Figure taken from Lovejoy et al. (2005).

produced by the process yet they are almost surely absent on any given realization. This means that they do not have the property of "ergodicity". What may be nothing more than normal multifractal statistical variability can thus easily be interpreted as breaks in the scaling. The second reason for erroneously concluding that the scaling is broken is the assumption that the scaling is isotropic. If the scaling is anisotropic, then breaks in the scaling on 1-D subspaces (transects) do not imply anything about the scaling of the full process.

\subsection{Apparent nonstationarity, inhomogeneity, parameter} variations... or simply random exponents?

When the statistical properties of a process are independent of spatial position, the process is statistically homogeneous; if independent in time, it is statistically stationary. These notions of statistical translational invariance are therefore properties of an infinite ensemble of realizations; indeed each realization will be inhomogeneous. In spite of this, the relatively low realization to realization inhomogeneity of the prevalent Gaussian or quasi Gaussian models (see e.g. Fig. 2, left hand column) has obscured this fact so that the existence of strong inhomogeneities - even if only on a single 


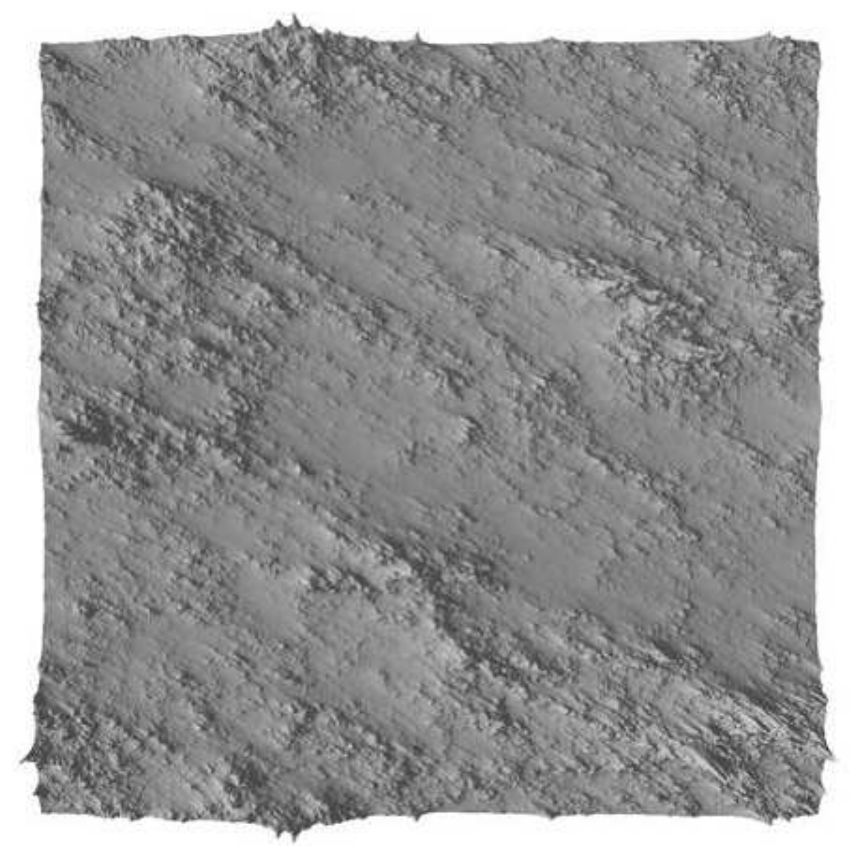

Fig. 5. $1024 \times 1024$ self-similar multifractal simulation with some trivial anisotropy and parameters $\alpha=1.9, C_{1}=0.12$ and $H=0.7$. The spectral exponent is $\beta=1+2 H-K(2)=2.17$.

realization - often leads to claims of statistical inhomogeneity/nonstationarity. In the case of multifractals, it is particularly tempting to invoke statistically inhomogeneous models (corresponding to different physical processes in different locations) since their occasionally strong singularities often stand out from a background of more homogeneous noise. However, the basic multifractal processes are statistically stationary/homogeneous in the strict sense that over the region over which they are defined (which is necessarily finite), the ensemble multifractal statistical properties are independent of the (space/time) location (and this, for any spectral slope $\beta$ ). Rather than discussing this at an abstract level, let us see what happens when we analyse a self-similar $1024 \times 1024$ multifractal simulation (Fig. 5). Figure 6 shows the compensated (i.e. $k^{2.17} E(k)$ ), isotropic spectrum obtained by integrating the Fourier modulus squared over circles of radius $k$ in Fourier space. The low frequencies are quite flat, indicating that the simulation has roughly the expected ensemble spectrum. At high frequencies, there is a drop-off which is an artifact of the numerical simulation techniques. We can now consider the "regional" variability in the spectral exponent $\beta$ by dividing the simulation into $8 \times 8$ squares, each with $128 \times 128$ pixels. Figure 7 (left) shows the histogram of the 64 regression estimates of the compensated spectra: the mean is close to zero as expected, but we see a large scatter implying that there are some individual regions having $\beta$ as low as 1.2 , some as high as 2.7 ; the standard deviation is \pm 0.3 . As we shall see later, this would imply a random

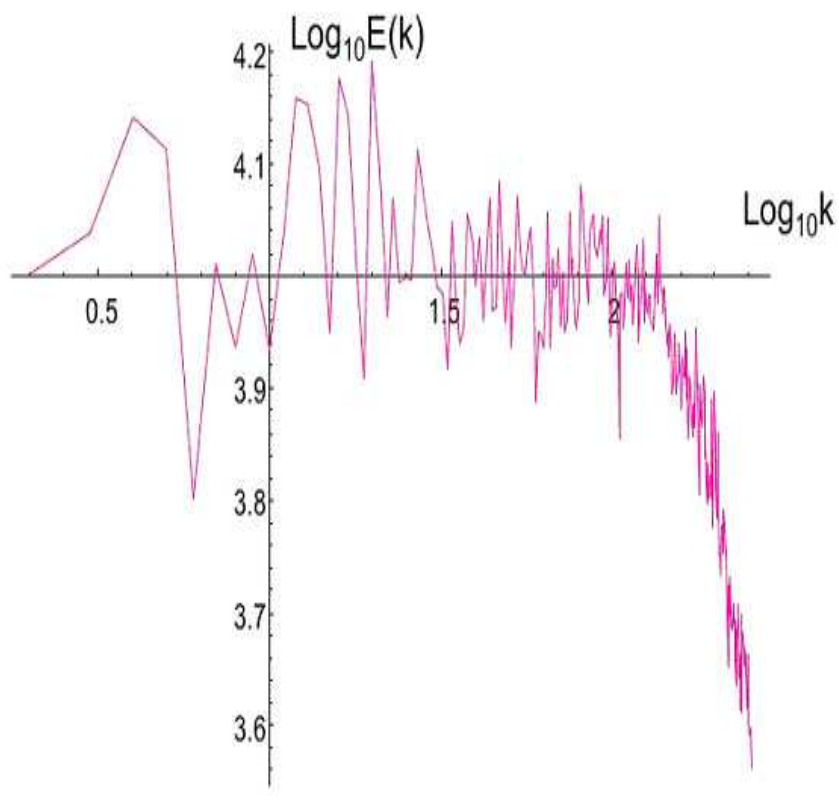

Fig. 6. Compensated power spectrum (i.e. $k^{2.17} E(k)$ ) of the multifractal simulation in Fig. 5. The extreme factor of 2 in wavenumber falls off too rapidly: this is an artifact due to the difficulty of discretizing singularities on numerical grids.
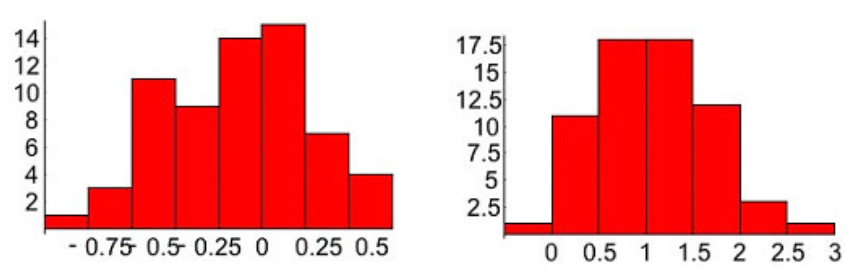

Fig. 7. On the left, we have a histogram of the compensated spectral exponent $\left(\Delta \beta=\beta-\beta_{\text {theo }}=\beta-2.17\right.$ ) values obtained after dividing Fig. 5 into $64128 \times 128$ squares, and computing the isotropic power spectrum in each square (the vertical axis is the number of occurences out of the total of 64). In each case, we fit the slope to the lowest factor of 16 in scale (we remove the highest factor of 4 due to numerical artifacts at the highest wavenumbers). On the right, we have a histogram of the $\log _{10} E_{1}$ ( $E_{1}$ is the spectral prefactor, $E(k)=E_{1} k^{-\beta}$ ) showing a variation of a factor of about 1000 from the smoothest to the roughest subregion.

variation in local estimates of $H$ of $\pm 0.3 / 2= \pm 0.15$, which is of the order of the difference observed between continents and oceans (see Sect. 6.1.1), although this spread in $\beta$ will decrease as the size of the data set increases. Similarly, use of the monofractal formula $D_{F}=7 / 2-\beta / 2$ would lead to a corresponding wide spread of "local" fractal dimension.

In Fig. 7, we can also see the large variations in the log prefactors (i.e. $\log _{10} E_{1}$, where $E(k)=E_{1} k^{-\beta}$ ). If this is interpreted in terms of roughness, the roughest of the 64 re- 
gions has about $10^{3}$ times the variance of the smoothest region. While it would obviously be tempting to give different interpretations to the parameters in each region, this would be a mistake. Note that this does not imply that the roughest and the smoothest would be associated with identical erosional, orographic or other processes. The point is that in a fully coupled model involving various geodynamical processes, all the processes would be scaling and would have correlated variations. Figure 4 also demonstrates the fact that if data from special locations (such as near high mountains) are analysed that we may expect systematic biases in our statistics and parameter estimates. These conditional statistics are discussed quantitatively and theoretically in Lovejoy et al. (2001b). This underlines the need for coupled multifractal processes, possible through the use of a state vector and vector mulitfractal processes (based on "Lie cascades" (Schertzer and Lovejoy, 1995; Lovejoy et al., 2001b)).

\section{Properties of multifractals}

\subsection{Multifractal processes and scale invariance}

In Sect. 2, we discussed the crucial role of singularities. Multifractals allow singularities of various orders to be distributed over fractal sets with varying fractal dimensions (rather than a unique dimension as for monofractal processes such as $\mathrm{fBm}, \mathrm{fLm})$. In this section we show how the key (multiplicative part) of the process can be viewed as a step by step build-up of variability from an initially uniform state. This multiplicative process is often called a "cascade"; it is the generic multifractal process.

Cascades are phenomenological models of processes which have the following properties: (1) They have a scale by scale conserved quantity; it is this conserved quantity that is modulated by nonlinear interactions as it goes down the scales. (2) Localness in Fourier space, i.e. structures of a certain size interact most strongly with structures of not too different sizes. (3) Scale invariance; over a range of scales, the mechanism doesn't change. To illustrate these points and show how cascades are related to multifractals, let us construct a very simple cascade, the $\alpha$-model (e.g., Schertzer and Lovejoy, 1985). This model is very close to the model of de Wisj (1951) for the distribution of mineral ores.

To help the reader through this section, a summary of our notation is provided in Table 2. Consider a certain quantity $\phi$ uniformly distributed in a $D$ dimensional space of size $L^{D}$ (to apply this to topography, just replace $D$ with 2). Supposing that the system (including its surroundings) has a very high number of degrees of freedom, we want to know how the quantity $\phi$ will be distributed in the $D$ dimensional space after undergoing a large number of nonlinear interactions with the whole system. Consider a simple discrete scale ratio model $(\alpha$-model); the continuous in scale extension is given in Table 1. The first step is to specify an integer ratio

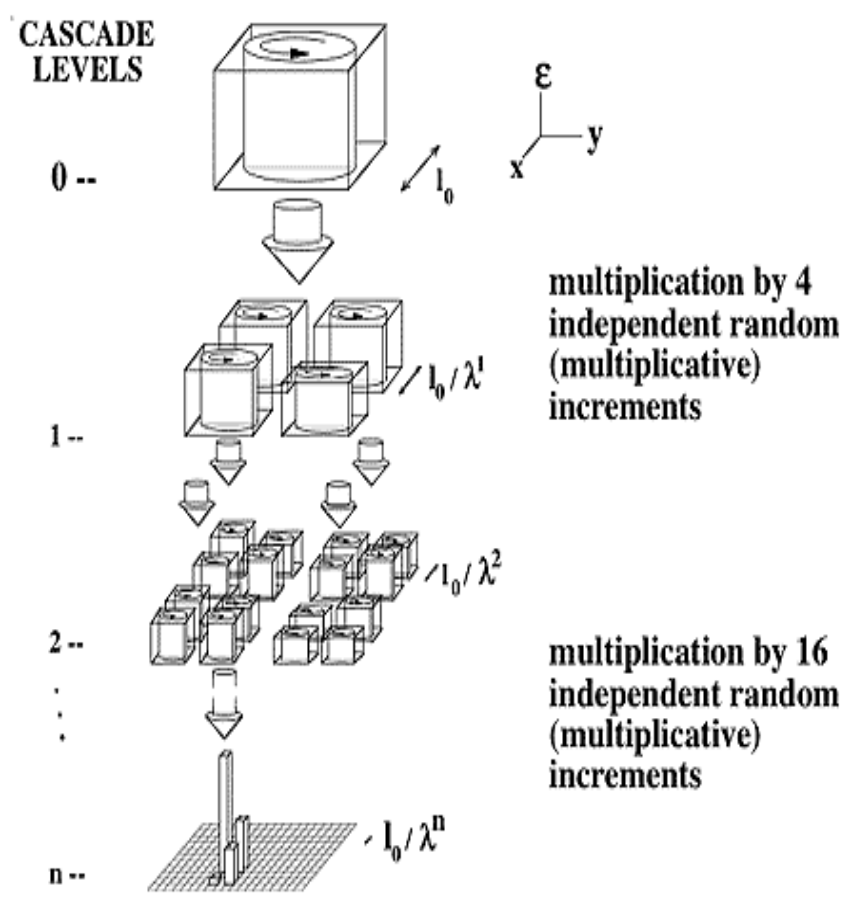

Fig. 8. Example of a 2-D cascade process. At each step of the cascade, the noise $\phi$ (represented by $\varepsilon$ in the above picture) in each square is multiplied by a random increment, here given by the probabilistic law in Eq. 5. Figure taken from Schertzer and Lovejoy (1985).

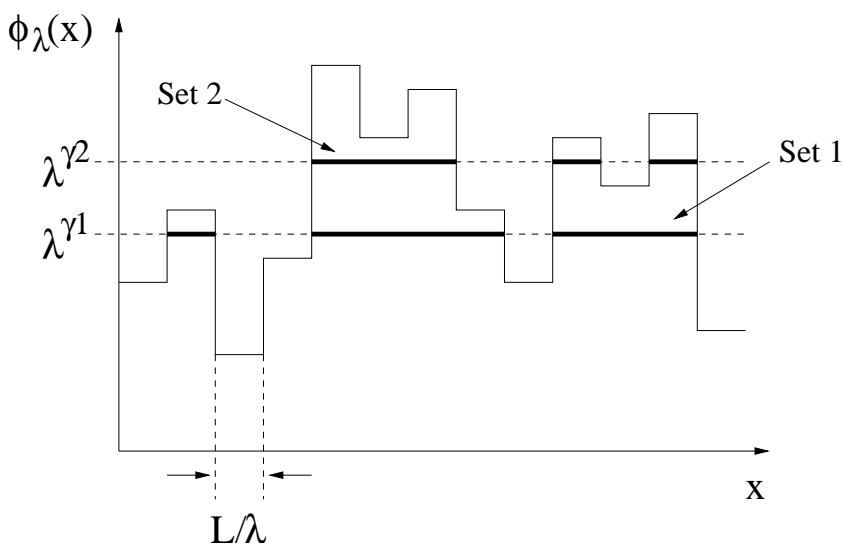

Fig. 9. Illustration of Eq. (6) using a 1-D example of a cascade. For different thresholds $t_{1}=\lambda \gamma_{1}$ and $t_{2}=\lambda \gamma_{2}$ (with $\gamma_{2}>\gamma_{1}$ ), the corresponding sets (defined by $\phi_{\lambda}>t$ ) have different fractal dimensions $\left(D_{F}\left(\gamma_{2}\right)<D_{F}\left(\gamma_{1}\right)\right)$ or fractal codimensions $\left(c\left(\gamma_{2}\right)>c\left(\gamma_{1}\right)\right)$.

of scales $\lambda_{0}=L / l_{0}$ (typically 2); we then separate the initial "cube" of size $L^{D}$ into $\lambda_{0}^{D}$ cubes of size $l_{0}^{D}$. In the $\alpha$-model, the quantity $\phi$ in each new cube at each step is modulated according to the following probabilistic law: 
Table 2. Summary of notation and important quantities.

\begin{tabular}{|c|c|c|c|}
\hline Symbol & Quantity & Definition & Reference $^{1}$ \\
\hline$l$ & Length scale & - & Eq. (6) \\
\hline$L$ & Maximum length of a process & - & Eq. (6) \\
\hline$\lambda$ & Resolution & $\lambda=L / l$ & Eq. (6) \\
\hline$T_{\lambda}$ & Scale changing operator & $T_{\lambda}=\lambda^{-G}$ & Eq. (2) \\
\hline$G$ & Generator & $D \times D$ matrix or operator & Eq. (2) \\
\hline$D$ & Dimension of space & - & Sect. 3.1 \\
\hline$D_{F}$ & Fractal dimension of a set & - & Sect. 3.1 \\
\hline$c$ & Codimension of a set & $c=D-D_{F}$ & Sect. 3.1 \\
\hline$\gamma$ & Order of singularity & - & Sect. 3.1 \\
\hline$t$ & Threshold & $t=\lambda \gamma$ & Fig. 9 \\
\hline$\phi_{\lambda}$ & Scaling multifractal noise & - & Sect. 3.1 \\
\hline$K(q)$ & Moment scaling function & $K(q)=\max _{q}\{q \gamma-c(\gamma)\}$ & Sect. 3.2 \\
\hline$\Delta h_{\lambda}$ & Height increment & $h\left(\mathbf{x}+\lambda^{-1} \Delta \mathbf{x}\right)-h(\mathbf{x})$ & Eq. (9) \\
\hline$\alpha$ & Degree of multifractality & $0 \leq \alpha \leq 2$ & Sect. 3.2 \\
\hline$C_{1}$ & Sparseness of the mean singularity & $0 \leq C_{1}$ & Sect. 3.2 \\
\hline$H$ & Degree of smoothing & - & Sect. 3.3 \\
\hline$\xi(q)$ & Structure function exponent & $\xi(q)=q H-K(q)$ & Sect. 3.3 \\
\hline$\beta$ & Spectral exponent & $\beta=1+\xi(2)$ & Sect. 5.1 \\
\hline
\end{tabular}

${ }^{1}$ Specifies the section or the equation where the symbol is defined or explained.

$$
\begin{aligned}
& \operatorname{Pr}\left(\mu \epsilon=\lambda_{0}^{\gamma_{+}}\right)=\lambda_{0}^{c} \\
& \operatorname{Pr}\left(\mu \epsilon=\lambda_{0}^{\gamma_{-}}\right)=1-\lambda_{0}^{c}
\end{aligned}
$$

where $\mu \epsilon$ is a "multiplicative increment" multiplying $\phi$, $\gamma_{+}>0, \gamma_{-}<0$ are positive/negative singularities and $c$ is the codimension of the space occupied by $\phi$. The de Wisj (1951) model is obtained by restricting this model so that the mean $\mu \epsilon=1$ on each cascade step (microcanonical conservation), whereas the above " $\alpha$-model" only enforces this conservation on the ensemble average (canonical conservation). It is now known that microcanonical conservation results in much less variable processes and is generally unrealistic. Equation (5) corresponds to an increase $\left(\lambda_{0}^{\gamma_{+}}\right)$or a decrease $\left(\lambda_{0}^{\gamma_{-}}\right)$ in $\phi$. Note that the parameters $\gamma_{+}$and $\gamma_{-}$cannot be chosen independently if we want $\phi$ to be conserved, as prescribed by assumption (1). After $n$ steps, $\phi$ is broken up into $\left(\lambda_{0}^{D}\right)^{n}$ cubes and the succession of decreases/increases leads to a highly heterogeneous $\phi$. In fact, it leads to a whole hierarchy of singularities $\gamma_{i}$ with values between $\gamma_{-}^{n} \leq \gamma_{i} \leq \gamma_{+}^{n}$ (see Fig. 8). The cascade just described is artificial because of its discrete in scale nature. Letting the scale ratio $\lambda_{0}$ become continuous (i.e. $\lambda_{0} \rightarrow 1 ; n \rightarrow \infty ; \lambda=\lambda_{0}^{n}=$ constant), we obtain (Schertzer and Lovejoy, 1987):

$\operatorname{Pr}\left(\phi_{\lambda} \geq \lambda^{\gamma}\right) \sim \lambda^{-c(\gamma)}$

where the notation $\phi_{\lambda}$ stands for $\phi$ at the scale $L / \lambda, \lambda \gamma$ is the threshold corresponding to the singularity $\gamma$ and $c(\gamma)$ is a nonlinear convex function called the codimension function. Equation (6) means that the set defined by the condition $\phi_{\lambda} \geq \lambda^{\gamma}$ has a (fractal) codimension given by
$c(\gamma)=D-D_{F}(\gamma)$, where $D_{F}(\gamma)$ is the fractal dimension of the set (see Fig. 9). Thus, the initial uniformly distributed quantity $\phi$ is now a hierarchy of interwoven sets, one for each threshold, each of them having a different fractal codimension: a multifractal. In comparison, a monofractal process has a unique fractal dimension for all thresholds. The scale by scale conserved multifractal $\phi_{\lambda}$ will be referred to as a multifractal noise.

\subsection{Moment scaling function and universal multifractals}

One way to characterize the statistics of stochastic processes is via probability distributions. For a multifractal process, the probability distribution is a power law, as given in Eq. (6). Equivalently, we can characterize the statistics of stochastic processes by the moments of the probability distribution $(\mathrm{Pr})$, $\left\langle x^{q}\right\rangle=\int_{0}^{\infty} d \operatorname{Pr} x^{q}$. In the case of multifractal processes, this gives (Schertzer and Lovejoy, 1987, 1991):

$\left\langle\phi_{\lambda}^{q}\right\rangle=\lambda^{K(q)}$

where $q$ is the order of the moment and $K(q)$ is a nonlinear convex function. $K(q)$ characterizes the scaling of the moments of the multifractal noise, hence it is called the "moment scaling function". If the multifractal noise is conserved with scale (i.e. $\left\langle\phi_{\lambda}\right\rangle=1$ ), then it implies that $K(1)=0$; for a nonzero process $K(0)$ is also trivially equal to zero. $K(q)$ and $c(\gamma)$ are related to each other via a Legendre transform, $K(q)=\max _{\gamma}\{q \gamma-c(\gamma)\}$. It is easy to see the equivalence between studying singularities $\gamma$ and moments $q$. In the case of singularities, we fix a threshold $\lambda^{\gamma}$ and then find the codimension $c(\gamma)$ of the set satisfying $\phi_{\lambda} \geq \lambda \gamma$. In the case of 
moments, we fix a moment $q$ and then take the average $\left\langle\phi_{\lambda}^{q}\right\rangle$; depending on $q$, certain orders of singularities dominate the average (for example, a high $q$ favors strong singularities compared to weaker ones). The bottom line is that analyzing a certain moment is equivalent to probing a certain singularity (a one to one correspondence given by the Legendre transform), but it is usually more convenient to do moments analysis.

A priori, the only constraint on $K(q)$ is that it must be convex, which implies that an infinite number of parameters are generally needed to describe it; without further constraints, it would not be manageable. Schertzer and Lovejoy (1987, 1991) have shown that there exists a class of stable and attractive multifractal processes called "universal multifractals", which are thus generic outcomes of multifractal processes independent of many of the details (see Schertzer and Lovejoy (1997) for the debate about this issue). Here we postulate the functional form given in Eq. (8) on theoretical grounds (it can be viewed as a consequence of a "multiplicative central limit theorem"), but we will see in Sect. 6.2.4 that the estimated error with this $K(q)$ is quite low, so that this can also be viewed as a way of parametrizing the data. The universal $K(q)$ is given by the following functional form:

$K(q)=\frac{C_{1}}{\alpha-1}\left(q^{\alpha}-q\right)$

where $\alpha$ and $C_{1}$ are the two basic parameters characterizing the scaling properties of the multifractal noise $\phi_{\lambda}$. The parameter $\alpha$ is the degree of multifractality and varies from 0 to 2 , where $\alpha=0$ is the monofractal case and $\alpha=2$ is the log-normal case. This parameter describes how rapidly the fractal dimension of sets at different thresholds vary as they leave the mean singularity. It is not very intuitive; the accompanying simulations (see Fig. 10) may be the best way to visualize the effect of varying $\alpha$. The parameter $C_{1}$ is the fractal codimension of the set giving the dominant contribution to the mean $(q=1)$ and is bounded below by zero. The value $C_{1}=0$ implies that the set giving the dominant contribution to the mean is space filling (i.e. its fractal dimension is equal to that of the embedding space), so it can be interpreted as quantifying the sparseness of the mean field. Again, compare Figs. $10-11$ or see the simulations of Fig. 12 to see the effect of varying $C_{1}$ on a multifractal process.

\subsection{Fractionally integrated flux model}

We have discussed (Table 1) several scaling models and have seen that $\mathrm{fBm} / \mathrm{fLm}$ are additive, being the result of a general scaling linear operator (a fractional integral/derivative) acting on a basic scaling noise (Gaussian and Levy, respectively). These lead to exponent functions linear in the moment $q$ (e.g. the structure function exponent $\xi(q)$, see Eq. (10)). We saw that in order to obtain more general scaling behaviour, we could retain the fractional integration of a noise, choosing the basic noise instead to be the result of a multiplicative process (the cascade). We saw that the cascade generates a scale by scale conserved noise $\phi_{\lambda}$ characterized by a moment scaling function $K(q)$ (a convex function with the constraints $K(0)=0, K(1)=0)$. The spectrum of the conserved noise has exponent $\beta=1-K(2)<1$. So to characterize topography (having $\beta \approx 2$ ), we clearly need an extra fractional integration; this is the Fractionally Integrated Flux model described in Table 1. The fractional integration of the multifractal noise leads to the following statistics for the height increments (Schertzer and Lovejoy, 1987, 1991):

$\Delta h_{\lambda}=\lambda^{-H} \phi_{\lambda}$

where $\Delta h_{\lambda} \equiv \Delta h(\Delta \mathbf{x})=h(\mathbf{x}+\Delta \mathbf{x})-h(\mathbf{x})($ with $|\Delta \mathbf{x}|=$ $L / \lambda)$ are the height fluctuations a distance $l=L / \lambda$ apart (as usual, the anisotropic generalization is obtained using $|\Delta \mathbf{x}| \rightarrow\|\Delta \mathbf{x}\|)$. The parameter $H$ can be interpreted as a degree of smoothness where higher $H$ means smoother fields. Figures 10-14 show examples of changing the degree of fractional integration $H$ in conjunction with $\alpha, C_{1}$.

As mentioned earlier, statistical moments analysis is more convenient than direct height analysis. We take the $q$ th power on both sides of Eq. (9) and then the ensemble average which leads to

$\left\langle\left|\Delta h_{\lambda}\right|^{q}\right\rangle=\lambda^{-\xi(q)} ; \xi(q)=q H-K(q)$

where $\left\langle\left|\Delta h_{\lambda}\right|^{q}\right\rangle$ is the $q$ th order structure function and $\xi(q)$ is the corresponding scaling exponent. The special case $q=2$ corresponds to ensemble averaged variograms. Equation (10) models the statistical properties of topographic height increments and is the culminating point of the multifractal FIF model. Note that if the multiscaling noise $\phi_{\lambda}$ in Eq. (10) is replaced with a noise with no particular relation between scales (i.e. $K(q)=0$ ) such as a Gaussian white noise, then we obtain fractional Brownian motion (see Table 1 for a comparison). This last comment is particularly important when it comes to data analysis and will help us to distinguish between multifractal and monofractal behavior (see Sect. 5.2). It also helps to clarify the advantage of multifractals over monofractals: the multifractal noise $\phi_{\lambda}$ is much more variable than Gaussian white noise, leading to a much better characterization of extreme events, such as very high mountains.

An important caveat is in order at this point. The 2-D analyses in this study are restricted to isotropic (i.e. self-similar) statistics. By isotropic analysis, we mean that the resolution $\lambda$ in real space is degraded isotropically or that the energy in Fourier space is integrated over wavevectors of constant length. In the example of the $\alpha$-model, one could break the $2-D$ noise $\phi$ in "rectangles" instead of "squares" to obtain an anisotropic cascade. The effect of using isotropic statistics is to wash out different geomorphologies/textures. As explained in Sect. 2.2, another scaling exponent $G$ - a matrix or, more generally, an operator - is needed to characterize scaling anisotropies, in addition to the usual $\alpha, C_{1}$, 

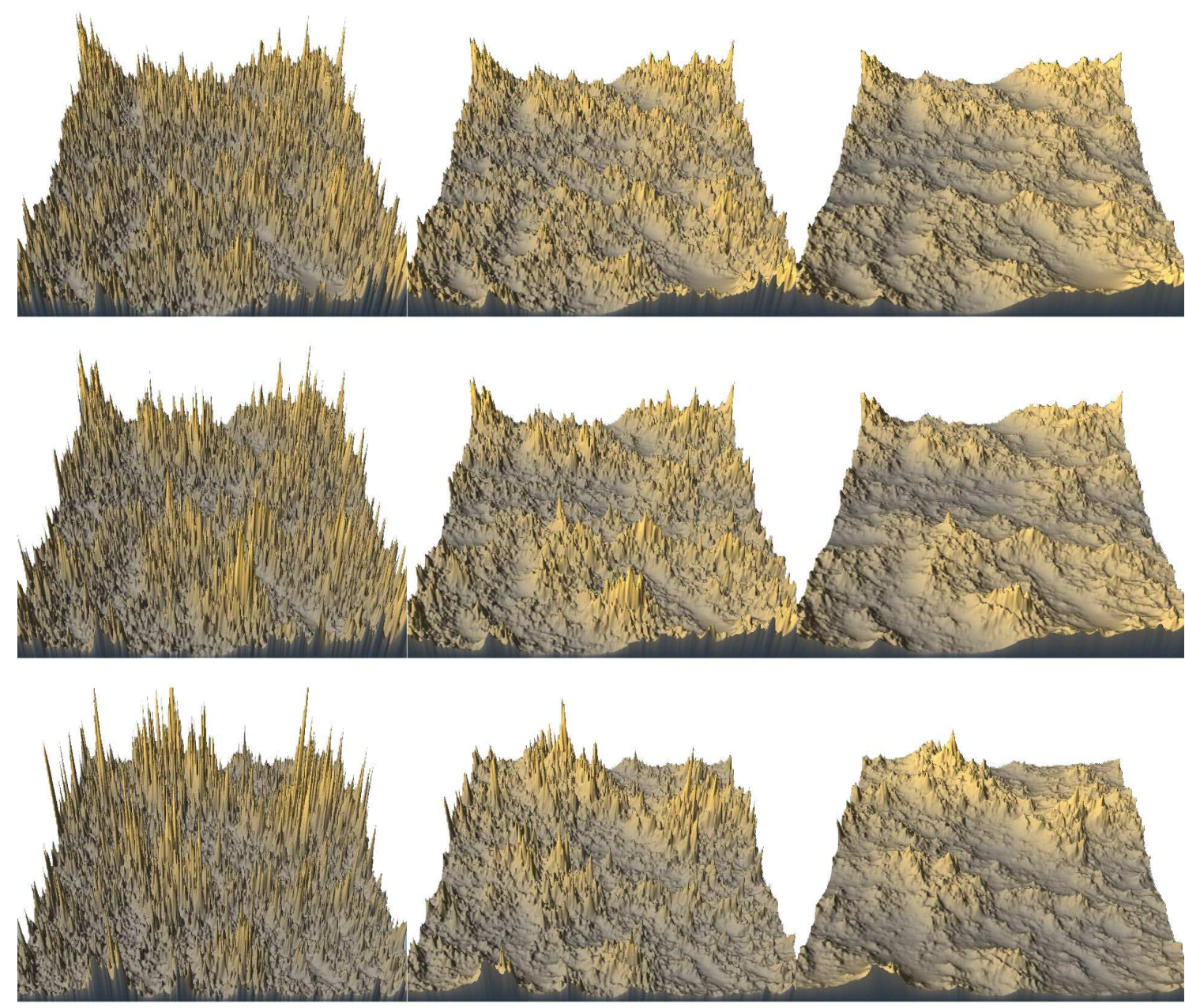

Fig. 10. Isotropic (i.e. self-similar) multifractal simulations showing the effect of varying the parameters $\alpha$ and $H$ ( $C_{1}=0.1$ in all cases). From left to right, $H=0.2,0.5$ and 0.8. From top to bottom, $\alpha=1.1,1.5$ and 1.8. As $H$ increases, the fields become smoother and as $\alpha$ decreases, one notices more and more prominent "holes" (i.e. low smooth regions). The realistic values for topography (see Table 5) correspond to the two lower right hand simulations. All the simulations have the same random seed.

$H$. We thus argue that isotropic statistics do not vary much from place to place even if morphologies/textures vary appreciably (see Figs. 2-3 and Figs. 12-14 for some isotropic vs anisotropic simulations). For additional simulations and "flybys" of topography and other geophysical fields, we refer the reader to the following website: http://www.physics.mcgill. $\mathrm{ca} / \sim$ gang/multifrac/index.htm. Additional simulations exploring anisotropic topographies may also be found in Lovejoy et al. (2005).

\section{Data sets}

In this study, several Digital Elevation Models (DEMs) that span various ranges of scales are analyzed. DEMs are gridded representations of topography. They are constructed via various techniques including stereo-photography and in situ measurements of altitude (often combined using complex objective analysis techniques) and are then gridded so as to obtain a height field. They are essentially characterized by their horizontal and vertical resolutions.

Due to their method of construction and the highly variable nature of the topographic field, DEMs have various limitations (e.g., Weissel et al., 1994), including insufficient dynamical ranges. The latter is defined as the ratio of the typical maximum signal to the noise level, which is usually determined by the vertical discretization. An insufficient dynamical range means that there is frequently not much pixel to pixel variability in the height measurements (i.e. adjacent pixels frequently have identical digital counts) so that gradients are not well represented, i.e. the surface is artificially smooth at the smallest spatial scales. We study this quan- 

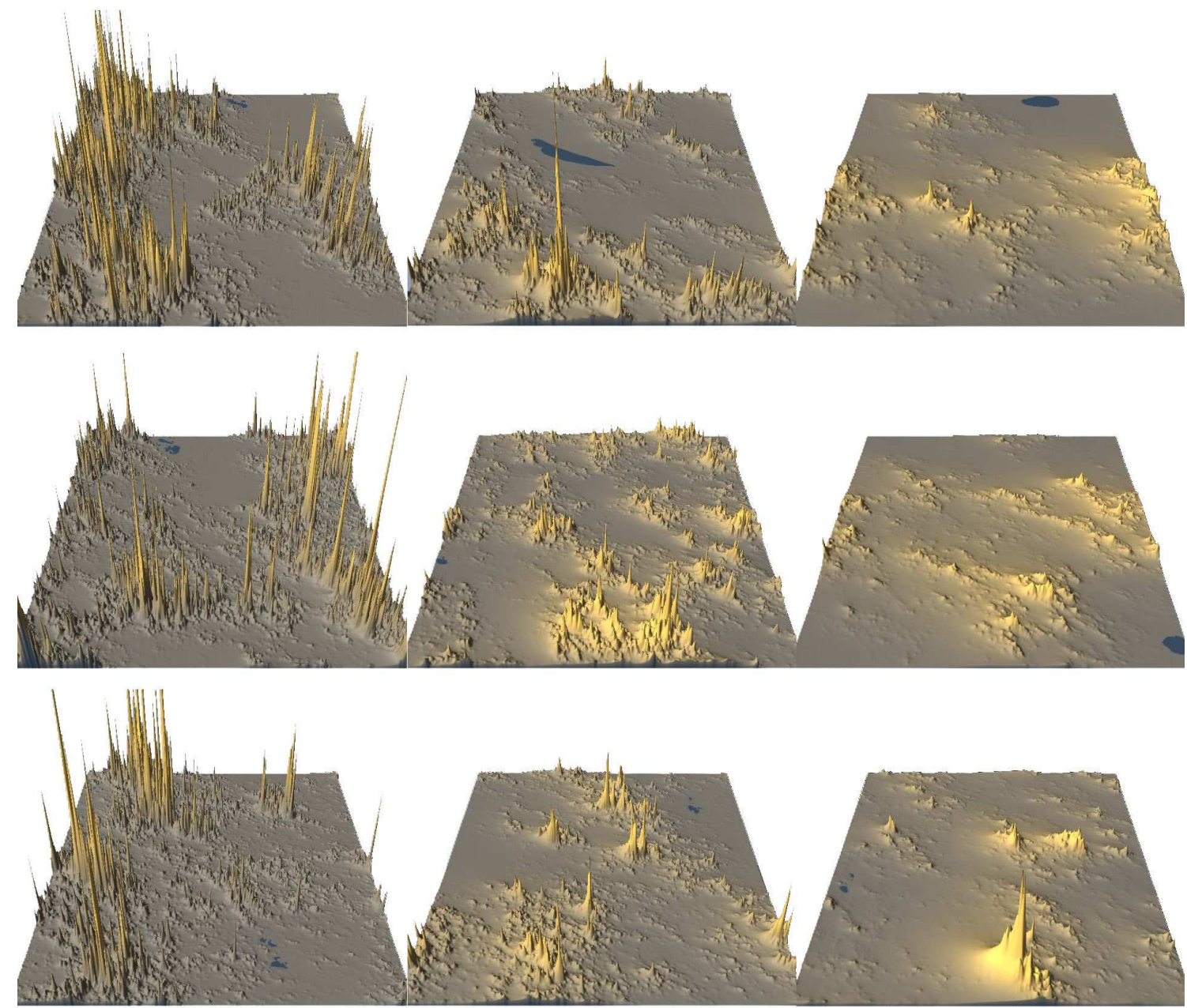

Fig. 11. Same as Fig. 10, but with $C_{1}=0.3$. The effect of increasing $C_{1}$ is to make high areas much more sparse. It is interesting to note the presence of isolated high peaks in very flat areas.

titatively in Sect. 6.2.2. Another problem is oversampling, which results when the altitude is sampled more frequently than is warranted by the source data; this implies an artificially smooth DEM at the highest wavenumbers. The curvature of the Earth can also be a problem; because DEMs are gridded representations of the topography, it is necessary to project a sphere on a plane to produce the DEM. When averaging many transects at different latitudes, this can induce changes in the highest wavenumbers. The main point is that each DEM has its own characteristics and problems, but these problems usually manifest themselves at the highest or smallest wavenumbers. On the other hand, different physical mechanisms would induce clean breaks in the scaling.

Four different DEMs are analyzed in this study. We refer the reader to Table 3 for their individual characteristics (resolution, vertical discretization, etc.) and to Figs. 15-17 for the regions analyzed on each of them. The data sets are:
ETOPO5 (global topography including bathymetry) (Data Announcement 88-MGG-02, 1988); GTOPO30 (global continental topography) (Land Processes Distributed Active Archive Center, 1996); United States (DEM of the United States) (United States Geological Survey, 1990); Lower Saxony (DEM of a $3 \mathrm{~km} \times 3 \mathrm{~km}$ section of Lower Saxony, constructed with the help of the High Resolution Stereo Camera Airborne (Wewel et al., 2000)).

\section{Analysis techniques}

In this section, we present two analysis techniques used to analyse scaling properties: power spectra and trace moments (a specific multiscaling analysis technique). In both techniques, ensemble averages are made over all available data. This is very important, because scale invariance is a statistical symmetry (see Sects. 2.3 and 2.4), meaning that an in- 

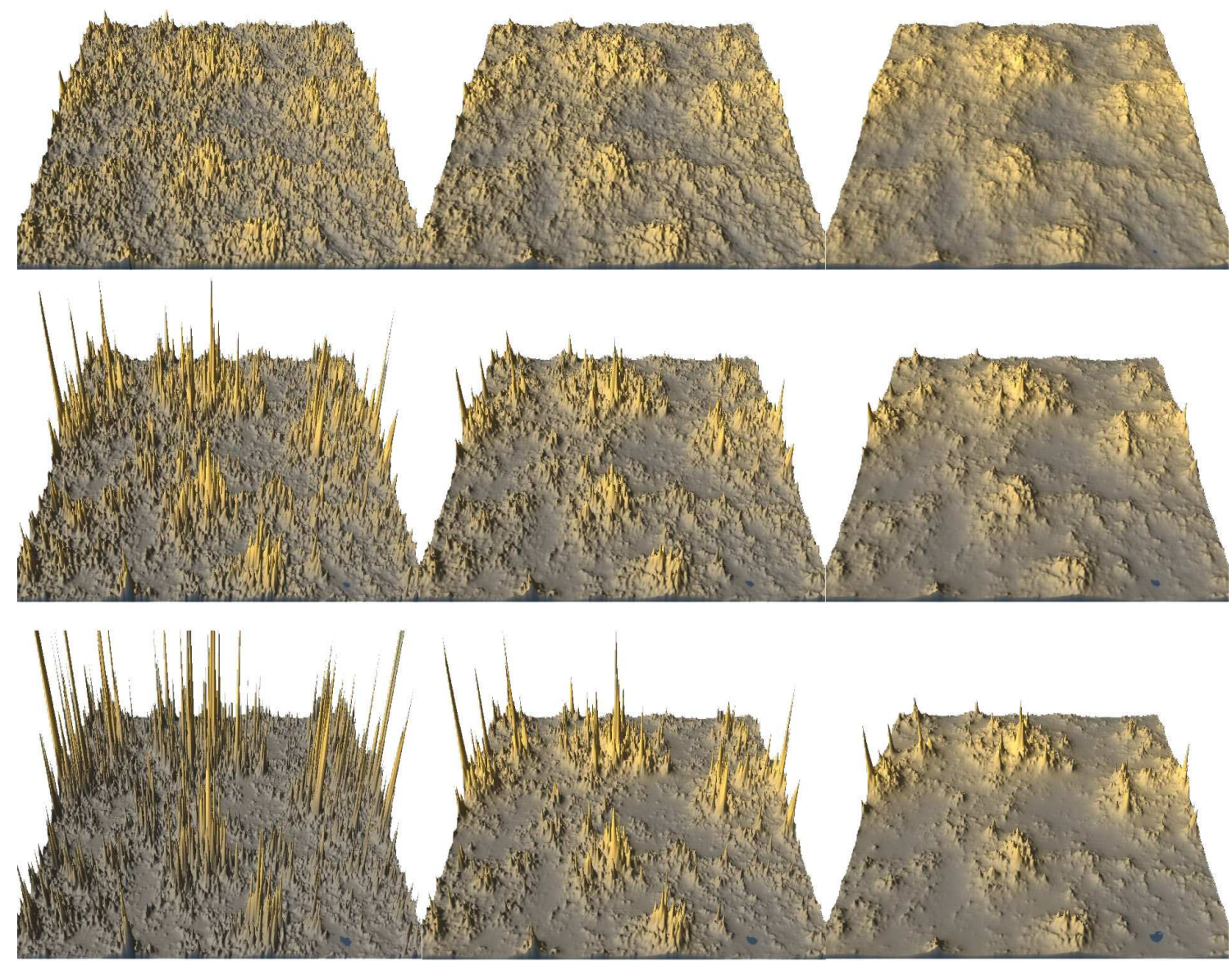

Fig. 12. Isotropic (i.e. self-similar) multifractal simulations showing the effect of varying the parameters $C_{1}$ and $H$ ( $\alpha=1.8$ in all cases). From left to right, $H=0.2,0.5$ and 0.8 . From top to bottom, $C_{1}=0.05,0.15$ and 0.25 . As $H$ increases, the fields become smoother. When $C_{1}$ is low, field values are close to the mean everywhere; when $C_{1}$ is large, all values are below the mean except in some specific locations where they are very large. The values closest to the data (see Table 5) correspond to the middle row (middle and right columns).

Table 3. Characteristics of the DEMs and regions studied.

\begin{tabular}{lcccc}
\hline Data sets & $\begin{array}{c}\text { Horizontal } \\
\text { resolution }\end{array}$ & $\begin{array}{c}\text { Vertical } \\
\text { discretization }\end{array}$ & $\begin{array}{c}\text { Numbers of } \\
\text { transects analyzed }\end{array}$ & $\begin{array}{c}\text { Length of } \\
\text { transects }(\mathrm{km})\end{array}$ \\
\hline ETOPO5 & $5^{\prime}(\approx 10 \mathrm{~km})$ & $1 \mathrm{~m}$ & 500 and ${ }^{1} 2160$ & 40000 \\
GTOPO30 & $30^{\prime \prime}(\approx 1 \mathrm{~km})$ & $1 \mathrm{~m}$ & 1225 & 4096 \\
U.S. & $90 \mathrm{~m}$ & $1 \mathrm{~m}$ & 2500 & 5898 \\
Lower Saxony & $50 \mathrm{~cm}$ & $10 \mathrm{~cm}$ & 3000 and $^{2} 500$ & 3 and $^{2} 0.512$ \\
\hline
\end{tabular}

1 Analysis with constant angular resolution.

2 Analysis on treeless region.

finity of independent samples are needed to obtain accurate scaling. In this study, all samples (transects or squares) analyzed are correlated because they come from the same region and from the same unique Earth. This means that the averages in this study are at best approximations to the required ensemble averages.

\subsection{Analysis of the height: Power spectra}

Power spectra are widely used to study scaling. In 1-D, it is the ensemble averaged squared modulus of the Fourier transform. In 2-D, it is usual to use the "isotropic" spectrum which is also angle integrated in Fourier space (sometimes 

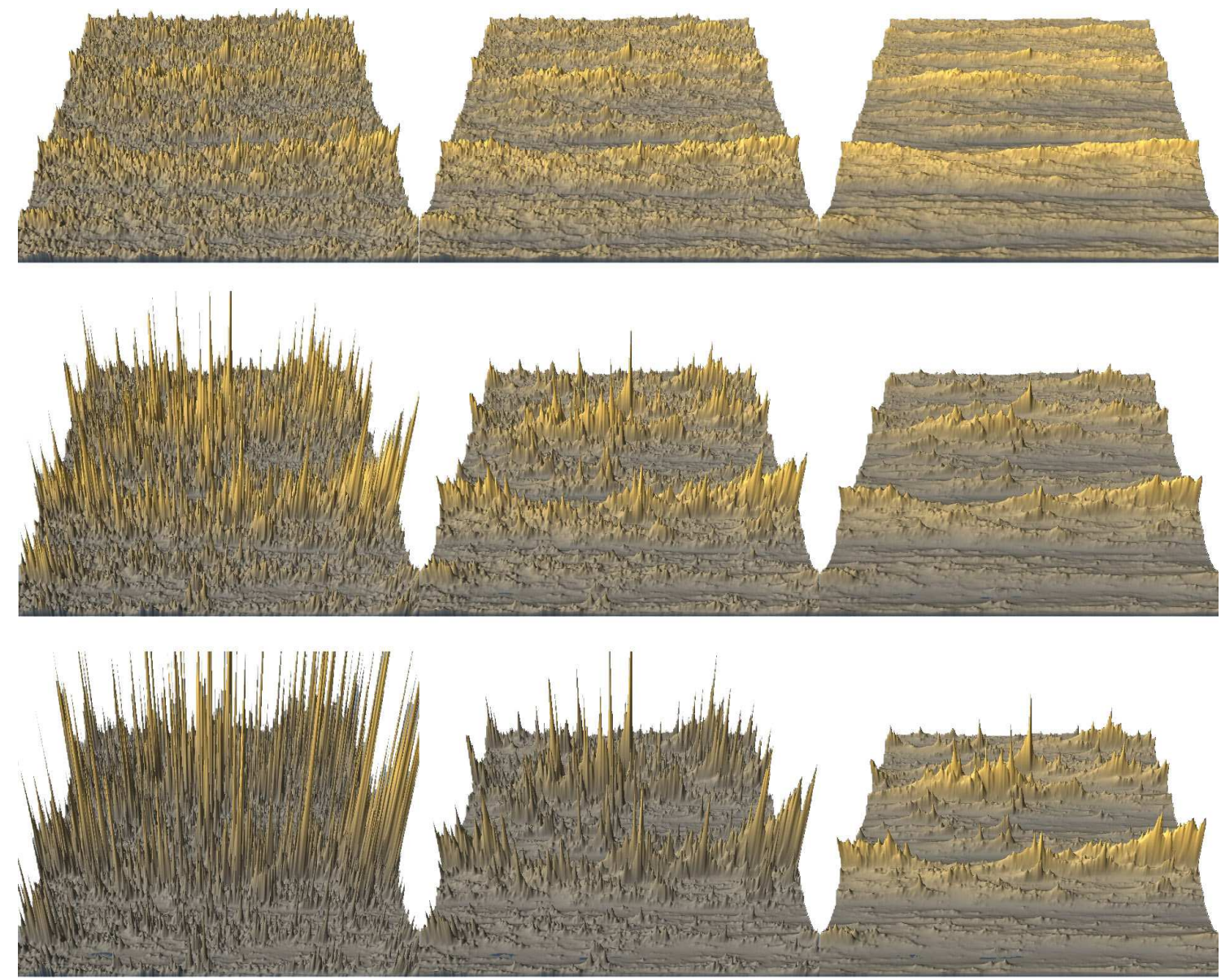

Fig. 13. Anisotropic (self-affine) multifractal simulations with varying $C_{1}$ and $H$ ( $\alpha=1.8$ in all cases). From left to right, $H=0.2,0.5$ and 0.8. From top to bottom, $C_{1}=0.05,0.15$ and 0.25 . The scale changing operator is $G=\left(\begin{array}{cc}H_{x} & 0 \\ 0 & H_{y}\end{array}\right)\left(\right.$ with $H_{x}=1.2$ and $\left.H_{y}=0.8\right)$ and the sphero-scale is 1 pixel. Transects in the up-down (y) or left-right (x) directions do not have the same spectral exponents: they are related through the ratio $\left(\beta_{x}-1\right) /\left(\beta_{y}-1\right)=H_{x} / H_{y}$, where $\beta_{x}, \beta_{y}$ are the 1-D spectral exponents in the $x, y$ directions, respectively.

angle averaging is used; this increases the spectral exponent by 1 ). Before estimating power spectra, we removed linear trends (in 1-D analyses) and we used Kaiser windows (in 2$\mathrm{D}$ in analyses) to avoid problems at the lowest wavenumbers. The (isotropic) power spectrum of a scaling process is given by

$$
E(\mathbf{k}) \propto|\mathbf{k}|^{-\beta}
$$

where $\mathbf{k}$ is the wavenumber and $\beta=1+\xi(2)=1+2 H-K(2)$ is the spectral exponent. It can be seen that the simple scaling result is recovered if $K(2)=0$. The power spectrum is only a second order moment, so that this method alone cannot be used to distinguish between simple and multiscaling.

\subsection{Analysis of the noise: Trace moments}

As can be seen from Table 1, the fBm and FIF models may be hard to distinguish because they both share a convolu- tion characterized by the exponent $H$. A better way to distinguish them is to consider their noise $\phi$, which has completely different properties: a delta correlated Gaussian white noise for fBm viz. a scaling singular multifractal noise for FIF. We are therefore lead to the use of "trace moments" (which directly characterize $\phi$ ) so that the distinction will be far more apparent. The first step is to obtain $\phi$ from the height increments: in principle this involves removing the $\lambda^{-H}$ in Eq. (9). From the convolutions in Table 1, we see that to invert the fractional integration, a fractional differentiation of order $H$ or greater must be performed (Schertzer and Lovejoy, 1987, 1991). For $H<1$, an adequate numerical approximation to this fractional differentiation is to take the modulus of the finite difference gradient (Lavallée et al., 1993), which corresponds to a differentiation of order $H=1$ (this is sufficient, because we have $H \approx 1 / 2$ in topography). More generally and equivalently, one can take the absolute 

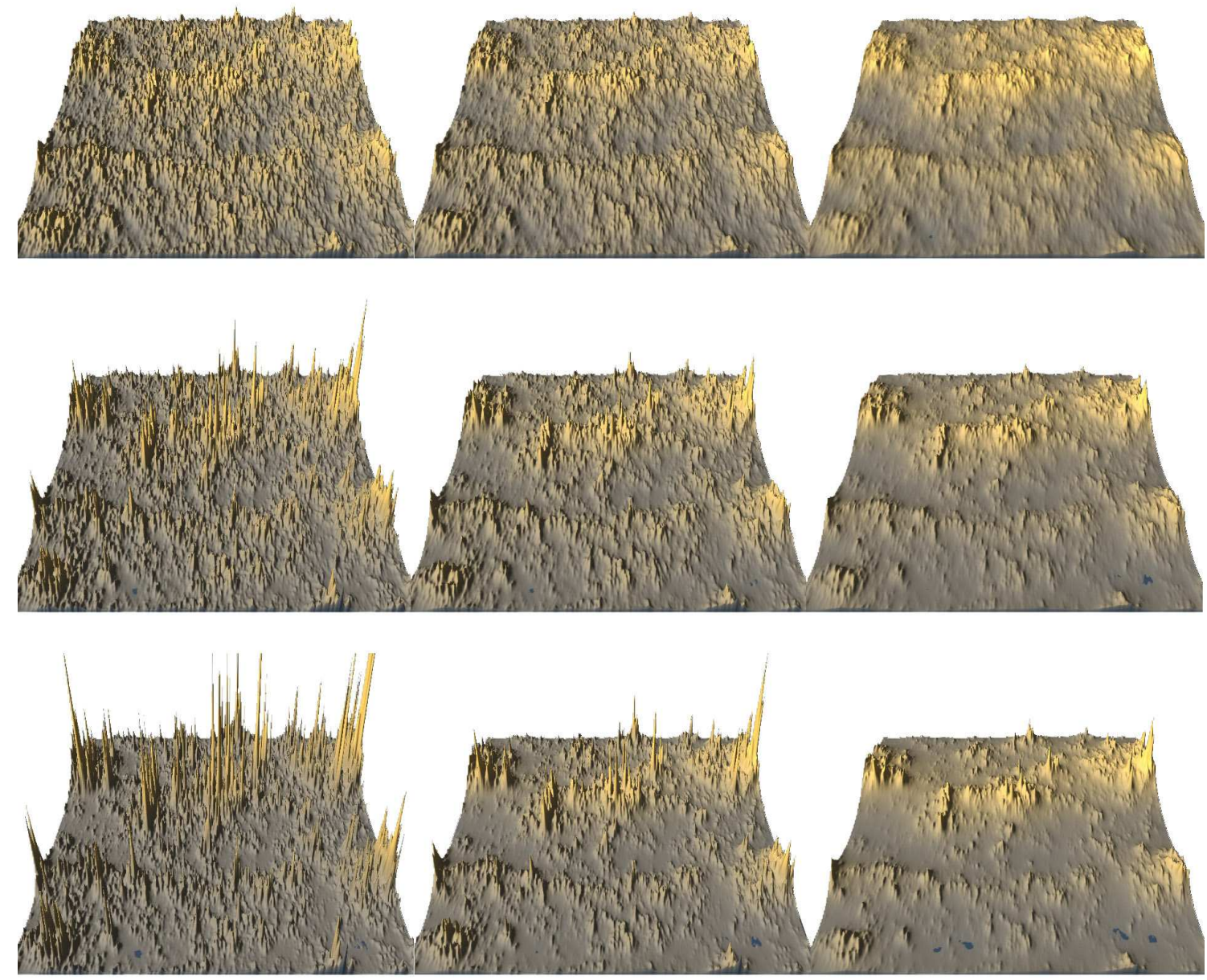

Fig. 14. Same simulations as Fig. 13, but with a sphero-scale equal to 64 pixels instead of 1 pixel. We still have left-right "mountain chains" but at scales smaller than 64 pixels, we have up-down "ridges". This is what a typical pixel of Fig. 13 would look like if blown up by a factor of 64 .

value of wavelet coefficients at the finest available resolution (Muzy et al., 1993; Audit et al., 2002). (Technical note: Because of the insufficient dynamical range of the DEMs (see Sect. 4), many spurious zero gradients are present in the analyzed transects. Those zero gradients particularly affect the low $q$ statistics, so they are eliminated by a fractional integration of order $H=0.1$ (a filtering in Fourier space with a power law), which is a scale invariant smoothing.) After the removal of $\lambda^{-H}$ in Eq. (9), we are left with only the underlying noise $\phi_{\lambda}$. The next step is to study the scaling of the statistical moments of $\phi_{\lambda}$ and compare them with Eq. (7). To do this, we normalize $\phi_{\lambda}$ so that the ensemble average of all the samples is $\left\langle\phi_{\lambda}\right\rangle=1$. Then spatial averaging is performed over sets (lines or squares) of size $l=L / \lambda$, the $q$ th power is taken and the average over all data available is taken: this gives the moments of the normalized noise for a given value of $q$. This procedure is performed for different values of $q$ and $K(q)$ is determined from the logarithmic slopes; multiscal- ing is verified if we find a nonlinear $K(q)$. For the monofractal $\mathrm{fBm}$, the moments of the normalized flux are equal to 1 for all $q$, i.e. $K(q)=0$. In other words, if after doing the fractional differentiation we get a Gaussian white noise (with no scale/resolution dependence, i.e. $K(q)=0)$, the data are compatible with a $\mathrm{fBm}$ process.

A recent article by Veneziano and Iacobellis (1999) points out that this method (i.e. taking the absolute value of the gradients before doing the trace moments analysis) may produce apparently spurious multiscaling. The reason is that taking the absolute value of the gradient is a nonlinear operation: it breaks the scaling at the highest resolution. However, we seek the scaling behavior of $\phi_{\lambda}$ at larger and larger scales (smaller and smaller $\lambda$ ); this scaling is rapidly re-established so that although it does take a certain range of scales (typically a factor of 2-4) before the averaged absolute gradients start to scale again, the exponent $K(q)$ can nonetheless be well estimated by this technique (all this has been extensively 
studied using numerical simulations (Lavallée et al., 1993). With the large topography data sets available in this study, the scale range is so large that this is not so difficult. In any case, functional box-counting (Lovejoy and Schertzer, 1990) and generalized structure functions (Lavallée et al., 1993; Weissel et al., 1994) also clearly indicate multiscaling over wide ranges of scale; breaks are indeed confined to the highest wavenumbers of each data set.

\section{Results and discussion}

\subsection{Analysis of continents and oceans}

The morphologies of continental and oceanic topography are clearly different. In the scaling framework, these differences can arise in several ways. One effect that we already mentioned (see Sect. 3.3) is that the anisotropy varies not only from scale to scale but also from place to place: another set of scaling exponents are needed to characterize these anisotropies. In this paper we limit ourselves to isotropic analyses (analysis of scaling anisotropy is quite difficult (e.g., Lewis et al., 1999)). For example, the 2-D spectra are angle integrated or in the case of trace moments we average on squares at each scale. In both cases, we "wash out" the anisotropies and consider only the isotropic statistics. We have also seen in Sect. 2 that due to their strong singularities, individual realizations of multifractals can have strong variations from one region to another even though the process is strictly homogeneous, stationary (statistically translationally invariant). This means that it is not trivial to test the scaling and estimate the parameters.

The continent/ocean comparison is made using power spectra and trace moments on ETOPO5. Three $512 \times 512$ pixels squares $(\approx 5120 \times 5120 \mathrm{~km})$ are analyzed in the case of continents and five in the case of oceans (shown on Fig. 15). The ensemble average is performed over the three (five) squares of continents (oceans) for the two methods of analysis.

\subsubsection{Power spectrum analysis}

A comparison between the averaged spectra from continents and oceans is shown on Fig. 18. According to Eq. (11), a $\log / \log$ plot of the spectral energy $E(k)$ versus the wavenumber $k$ should give a straight line if the process is scaling. The spectra are fairly straight over 2 orders of magnitude, implying that they are scaling over that range. There is a break in the scaling at approximately $50 \mathrm{~km}$, which is probably due to oversampling. Note that there is a systematic difference in the slope of the spectra: $\beta_{\text {continents }}=2.09$ (c.f. $\beta \approx 2$ (Vening Meinesz, 1951)) and $\beta_{\text {oceans }}=1.63$, in agreement with Berkson and Matthews $(1983)(\beta \approx 1.6-1.8)$ but less than the values of Bell $(1975)(\beta \approx 2)$, Fox and Hayes $(1985)(\beta \approx 2.5)$ and Gibert and Courtillot (1987) $(\beta \approx 2.1-2.3)$. The differences between the values of $\beta$ may be a consequence of

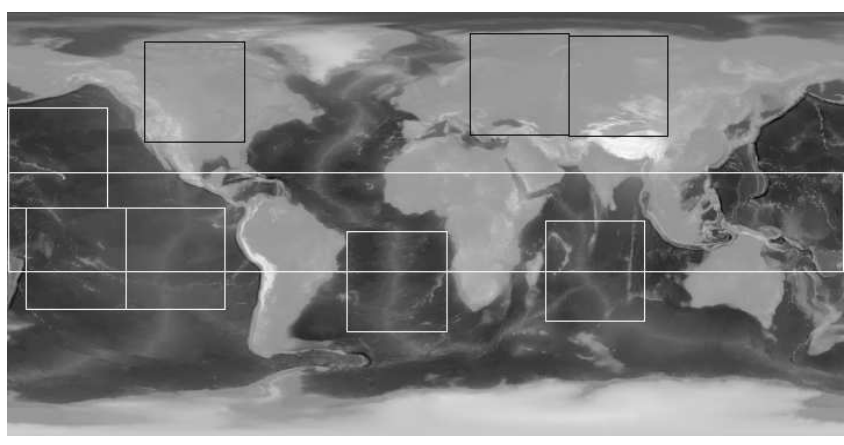

Fig. 15. ETOPO5 data set (Data Announcement 88-MGG-02, 1988). See Table 3 for its specific properties. The white and black squares indicate the areas studied in Sect. 6.1. The white rectangle indicates the area used for the narrow strip analysis (500 transects of $40000 \mathrm{~km})$.

the fact that estimates of the spectral exponent require large amounts of data and wide ranges of scales (see Sect. 2.3). In addition, for multifractals the values of $\beta$ in any single realization is random with (depending on parameters) a possibly large scatter (see Sect. 2.4). In the studies mentioned above, the analyses are on small data sets $\left(10^{4}\right.$ compared to $10^{6}$ points here), which result in "fuzzy" or even broken spectra, making it hard to find the scaling range. This means that the spectral exponents may not be well estimated in those studies.

6.1.2 Trace moments analysis and the universal multifractal parameters

The trace moments of the continents and the oceans are shown in Fig. 19. According to Eq. (7), a log/log plot of the normalized moments of the noise $\phi_{\lambda}$ versus $\lambda$ should give straight lines (with different slopes for each $q$ ) if the process is multiscaling. As can be seen on Fig. 19, the trace moments are straight for scales greater than $50 \mathrm{~km}$; as with the spectra, they flatten a little at small scales, presumably for the same reason (probably a little oversampling). However, we see clearly that the behavior is totally different from that predicted by the monofractal $\mathrm{fBm}$ model; in the latter, all lines would be quite flat, there would be no systematic resolution dependence. The multifractality stands out as a systematic roughly linear trend with nonzero slope. Indeed, the standard errors of the slope indicate that for typical values of $q$, the slope is $5-10$ standard deviations away from the fBm value zero, so that the $\mathrm{fBm}$ hypothesis can be easily rejected with standard statistical tests. We could mention at this point that although it has never been proposed for the topography, there exists a monofractal model for the multiplicative noise called the " $\beta$-model" in which $\phi$ is confined to a fractal support (this corresponds to $\alpha=0$, see Sect. 3.2). The $\beta$-model is quite different from $\mathrm{fBm}$ since the latter has a nonfractal (space 


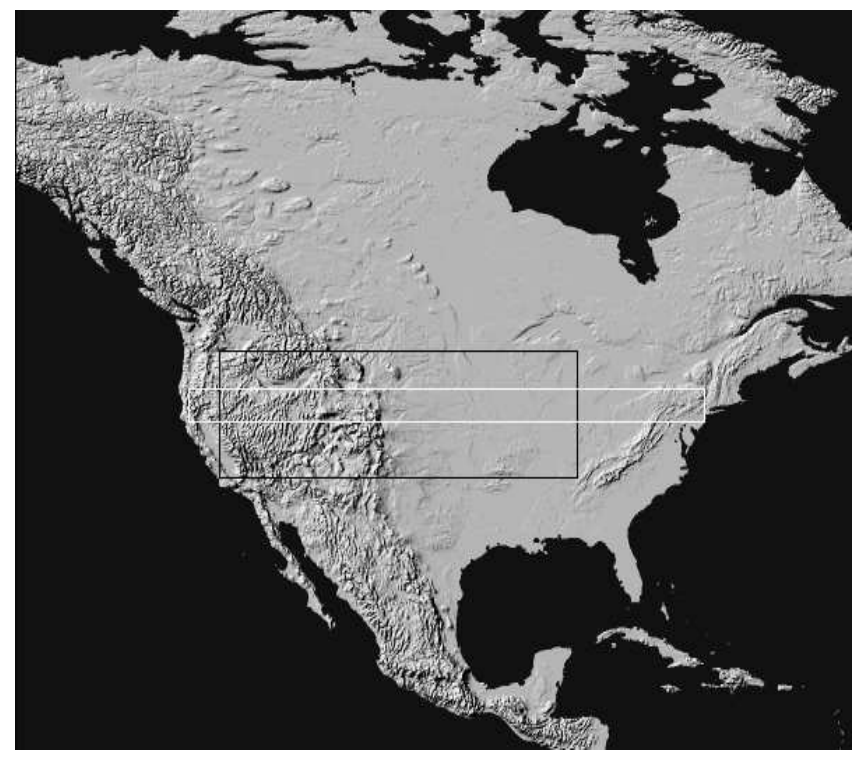

Fig. 16. U.S. part of the GTOPO30 data set (Land Processes Distributed Active Archive Center, 1996). See Table 3 for its specific properties. The black rectangle indicates the area studied on GTOPO30 (1225 transects of $4096 \mathrm{~km}$ ) and the white rectangle indicates the area studied on the U.S. DEM (2500 transects of $5898 \mathrm{~km})$.

Table 4. Universal multifractal parameters for the continents/oceans analysis.

\begin{tabular}{lccc}
\hline Type of region & $\alpha$ & $C_{1}$ & $H$ \\
\hline Continents & $1.82 \pm 0.17$ & $0.13 \pm 0.04$ & $0.66 \pm 0.04$ \\
Oceans & $1.87 \pm 0.15$ & $0.15 \pm 0.02$ & $0.46 \pm 0.03$ \\
Continental margins & $1.80 \pm 0.05$ & $0.12 \pm 0.02$ & $0.77 \pm 0.02$ \\
\hline
\end{tabular}

filling) support corresponding to $C_{1}=0$. In Fig. 19, the $\beta$-model would give nonzero slopes that would vary linearly with $q$ (the $K(q)$ 's in Fig. 20 would follow $K(q)=C_{1}(q-1)$ ). We see below that this is also incompatible with the data. We may also note that the fLm is not compatible with the data either; this is because statistical moments substantially larger that $q=2$ have no obvious anomalous behaviours (visible as a "multifractal phase transition", i.e. a discontinuity in the derivative of $K(q)$ at $q=2$ ).

The slopes of the trace moments for each $q$ yield $K(q)$; from this we estimate the parameters $\alpha$ and $C_{1}$ for continents and oceans. Equation (8) is used to fit the curves on Fig. 20 and the results are shown in Table 4. The errors in the table are equal to one standard deviation of the individual values of $\alpha$ and $C_{1}$ for continents and oceans. By taking into account the error, we conclude that continents and oceans may have the same $\alpha$ and $C_{1}$.

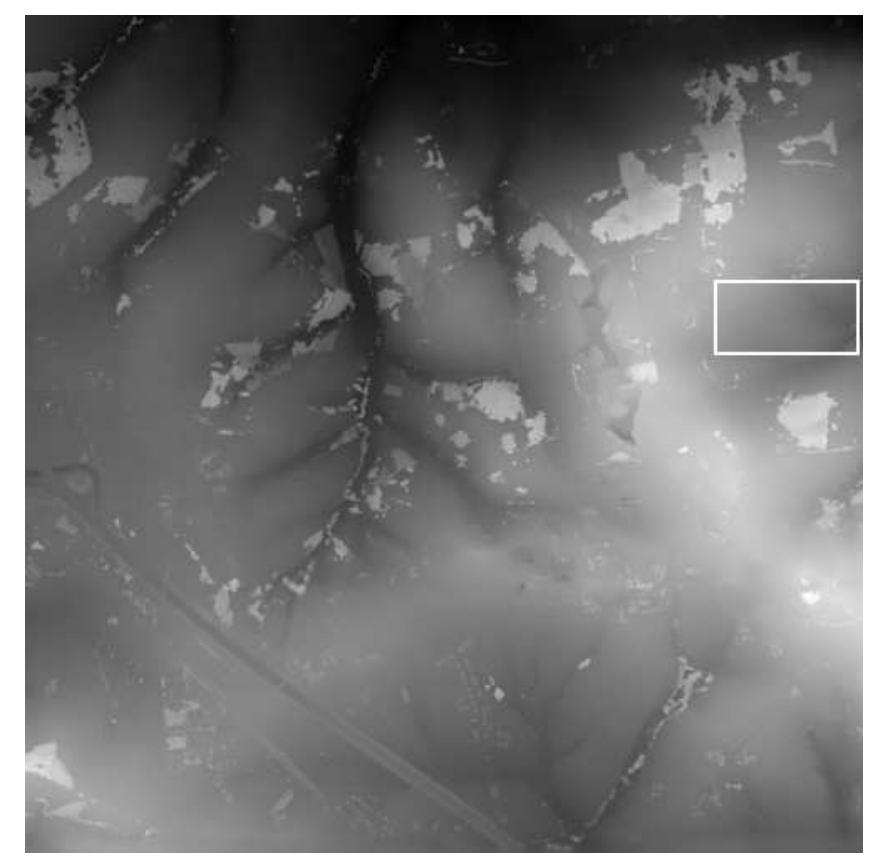

Fig. 17. Lower Saxony DEM (Wewel et al., 2000). See Table 3 for its specific properties. The global analysis of Sect. 6.2 is done on the upper half of the above DEM to avoid contamination from man-made structures. The total area covered is 3000 transects of $3000 \mathrm{~m}$. The small white rectangle (500 transects of $512 \mathrm{~m}$ ) is the treeless section analyzed and compared to the full DEM analysis.

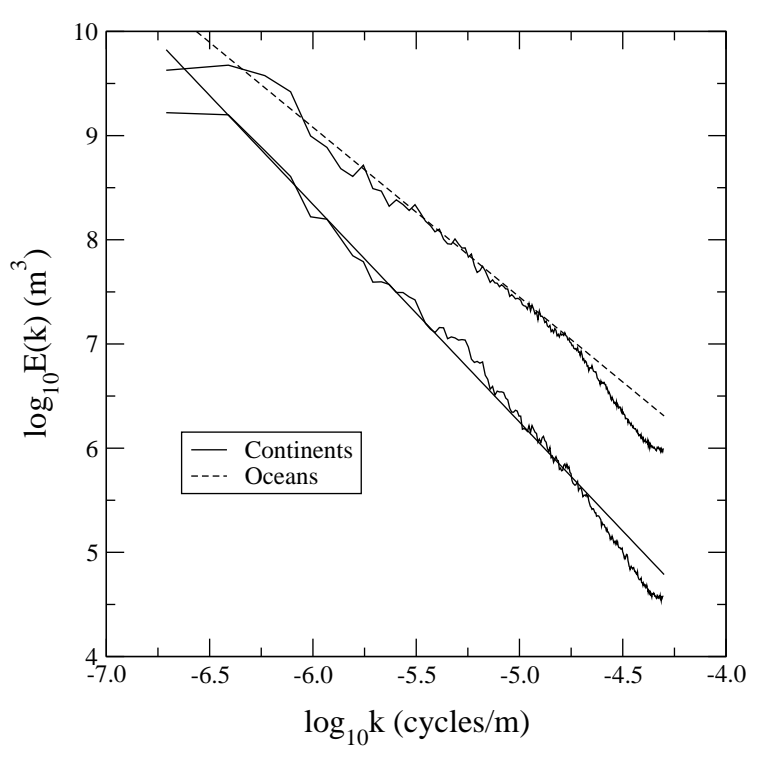

Fig. 18. $\mathrm{Log} / \log$ plot of the spectral energy versus the wavenumber for continents and oceans. The slopes $(=-\beta)$ are -2.09 for continents and -1.63 for oceans. 

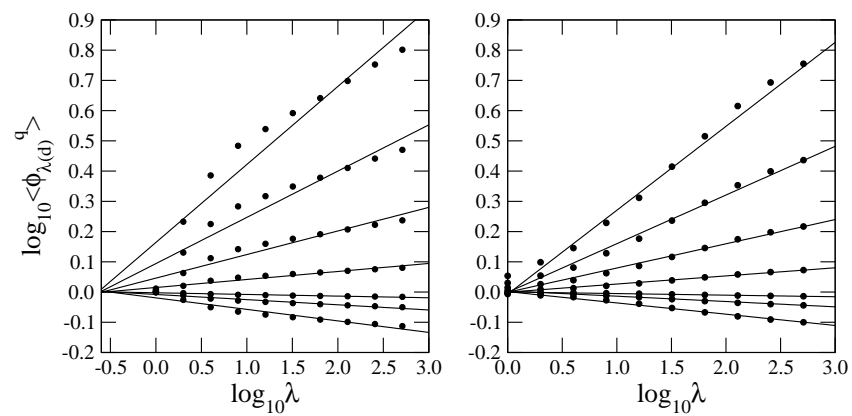

Fig. 19. $\log / \log$ plot of the normalized trace moments versus the scale ratio $\lambda=L / l$ for continents (left) and oceans (right), both with $L=5120 \mathrm{~km}$. The values of the exponent $q$ of each trace moments are, from top to bottom, 2.18, 1.77, 1.44, 1.17, 0.04, 0.12 and 0.51.

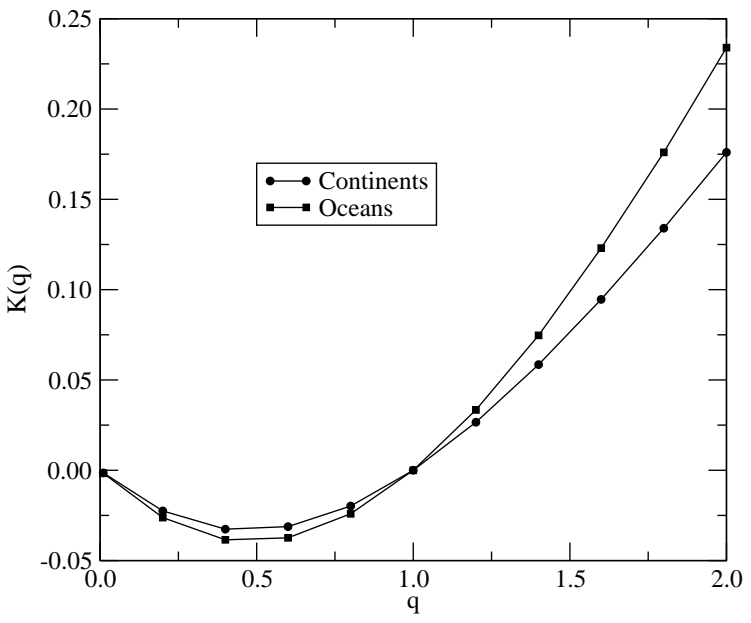

Fig. 20. Plot of the moment scaling function $K(q)$ as a function of the moment $q$ (circles correspond to continents and squares to oceans)

The values of $\alpha$ and $C_{1}$ combined with $\beta$ yield the values of $H$ for continents and oceans (see Eq. 11 and following discussion). The results are shown in Table 4 . The $H$ value for oceans is quite near the commonly cited value of $H=1 / 2$ (e.g., Bell, 1975), but the $H$ value for continents is systematically larger. This can also be seen on the scatter plots of Figs. 21 and 22, where there is a clear stratification of the $H$ values but not of the $\alpha$ and $C_{1}$ parameters (where there is only a certain spread). This result is compatible with the hypothesis that the statistics of the noise $\phi_{\lambda}$ (determined by $\alpha$ and $C_{1}$ ) are the same for continents and oceans, but the height statistics (see Eqs. 7 and 10) are different because $H$ is different. The higher value of $H$ for continents means that they are smoother than the seafloor. It means that the underlying physical mechanisms responsible for the values of $\alpha$ and $C_{1}$, whatever they are, may be the same for continents

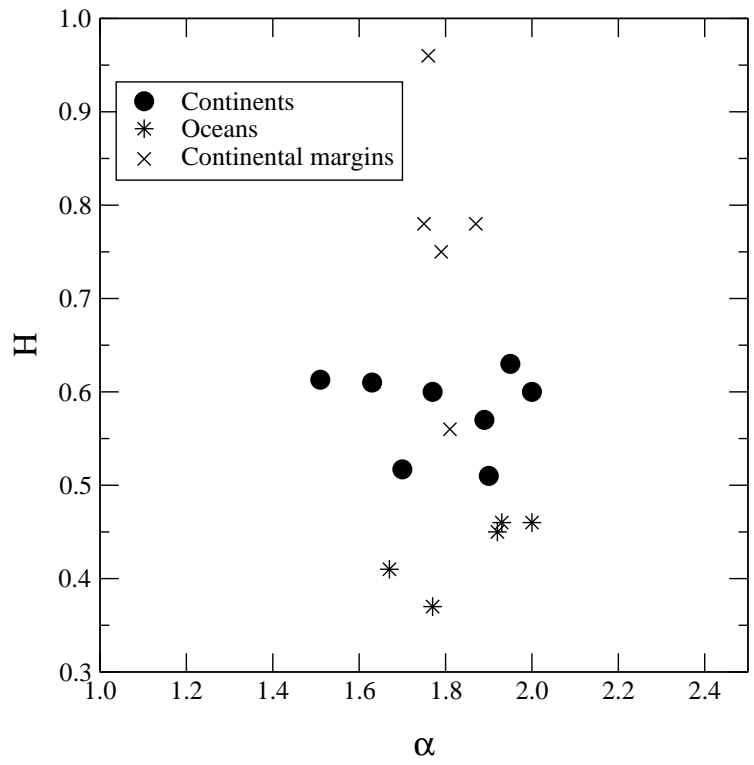

Fig. 21. Scatter plot of the parameters $(\alpha, H)$ for the three different types of region (circles, stars and X's represent continents, oceans and continental margins respectively).

and oceans. On the other hand, the mechanisms responsible for $H$ (related to erosion-like processes) are likely to be different. It also means that the simple dimensional analysis (with $H=1 / 2$ ) proposed by Lovejoy et al. (1995) does not hold on continents.

\subsubsection{Continental margins}

In addition to the continents and oceans, it is also of interest to consider the statistics of the transition region from one to the other, i.e. the continental "margins". These regions have very large topographic gradients and may therefore have a different $H$. Also, since Sect. 6.2 deals with the global analysis of topography, i.e. the combined analysis of continents and oceans (on the same transect), this region is important. So we apply the same procedure as for continents and oceans on 5 square regions that include a continental margin (not shown on Fig. 15, but very near the squares already drawn). The final universal parameters are given in Table 4. As can be seen, once again the $\alpha$ and $C_{1}$ parameters do not differ significantly from those of continents and oceans, but the $H$ parameter is significantly higher. This can also be seen from the scatter plots (Figs. 21 and 22). It is not clear what is the physical explanation for this higher value of $H$ for continental margins, except that it implies a smoother variation from high to low altitudes. Since the algorithms used to interpolate between continents and oceans smooth the data, we should be cautious in our interpretation of this result. 


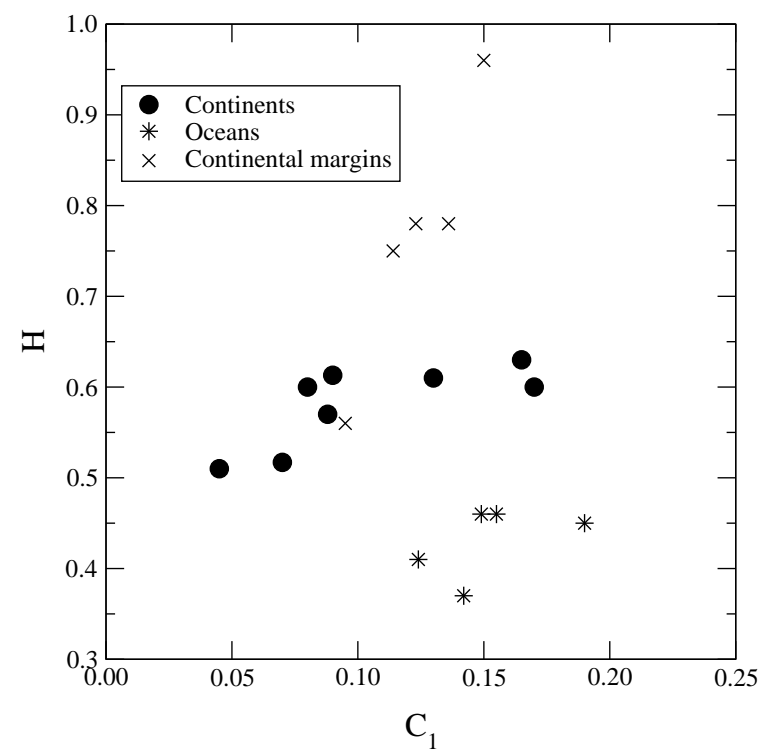

Fig. 22. Scatter plot of the parameters $\left(C_{1}, H\right)$ for the three different types of region (circles, stars and X's represent continents, oceans and continental margins respectively).

\subsection{Global topography analysis}

In this section, we use power spectra and trace moments to analyze the four DEMs. For convenience, the data are analyzed in 1-D (transects) that are chosen so as to maximize the range of scales analyzed (i.e. we take the longest possible transects on each DEM). The regions analyzed are shown on Figs. 15 (ETOPO5), 16 (GTOPO30 and the U.S. DEM) and 17 (Lower Saxony DEM). See Table 3 for details. In the initial analysis of ETOPO5, only 500 transects from a narrow strip around the equator are used. This is done in order to obtain a relatively constant spatial resolution (the original DEM has constant angular resolution), so that it can be compared with the results of the other DEMs. Below, we also analyze the ETOPO5 data as a function of angular resolution (using all the 2160 transects of the data set).

\subsubsection{Power spectrum}

Figure 23 shows the results of the power spectrum analysis over the four DEMs (the spectrum is on the narrow strip for ETOPO5). The $\log / \log$ plots on Fig. 23 give straight lines over 6 orders of magnitude (the individual values of $\beta$ are given in Table 5), indicating that the scaling is well respected from planetary scales down to a few meters. In fact at $40 \mathrm{~m}$, there is a "bump" that breaks the scaling in the Lower Saxony spectrum. The same break in the scaling is also observed in the trace moments analysis (see Sect. 6.2.4). This break can probably be explained by the presence of trees (irregular white patches on Fig. 17) on the Lower Saxony DEM (Gagnon, 2001). Below we will concentrate on a tree-free

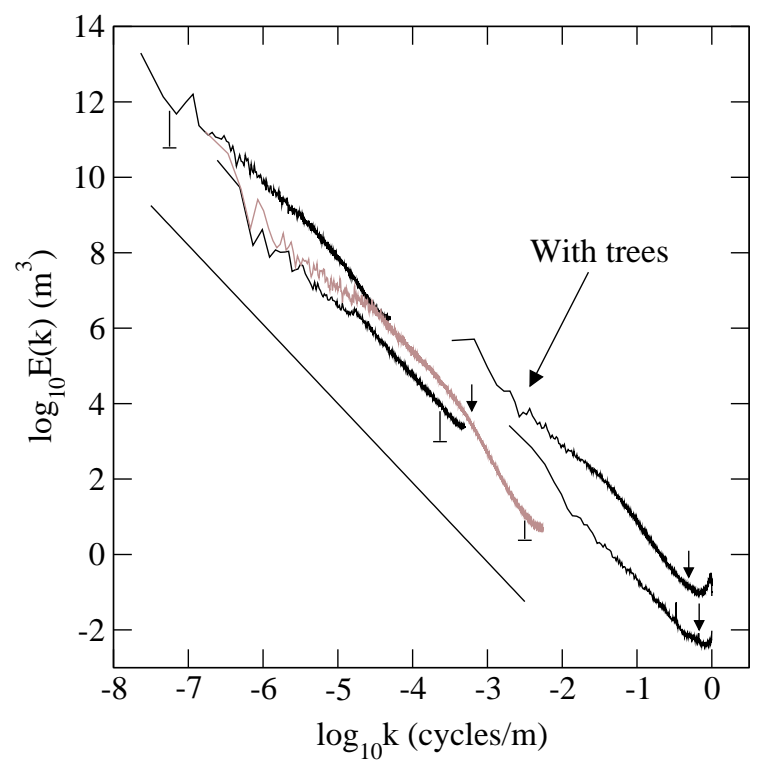

Fig. 23. $\log / \log$ plot of the spectral energy versus the wavenumber for the four DEMs. From right to left: Lower Saxony (with trees, top), Lower Saxony (without trees, bottom), U.S. (in grey), GTOPO30 and ETOPO5. A reference line of slope -2.10 is on the graph to show the overall slope of the spectra. The small arrows show the frequency at which the spectra are not well estimated, according to Eq. (13) (for ETOPO5, it is well estimated on the whole range). The "semi error bar" symbols indicate the amount of offset due to the resolution dependent factor $\lambda^{K(2)}$ (see Eq. 14 for details). Figure taken from Gagnon et al. (2003) with some modifications.

part of the DEM to avoid problems (indicated on Fig. 17). Its power spectrum is also shown on Fig. 23: we can see that it follows the "overall" spectrum, hinting at scaling down to the meter scale. The fact that the spectrum containing trees is shifted vertically compared to the tree-free one is easily explained. Trees are not part of natural topography, they are superposed on top of it; they thus add spectral energy to the spectrum, resulting in a vertical shift. The spectrum of the full ETOPO5 data set is given in Fig. 24. It is straight over $\approx 2.7$ orders of magnitude and the value of $\beta$ is given in Table 5. We can see that the values of $\beta$ are similar for the narrow strip analysis and the full analysis.

\subsubsection{Quantitative analysis of insufficient dynamical range}

Before interpreting the data further, we must consider the effects of artefacts due to the inadequate dynamical range of the DEMs. The following rough argument can be used to find the wavenumber $k_{\max }$ at which the spectrum starts to be corrupted by this effect. Let $\Delta h_{\max }=h_{\max }-h_{\min }$ be the maximum range of altitude on the data set and $\Delta h_{\min }$ the smallest possible nonzero difference on the data set (which is equal to the height of one unit of vertical discretization). The ratio $\Delta h_{\max } / \Delta h_{\min }$ defines the dynamical range of the 
Table 5. Universal multifractal parameters for the four DEMs (and other studies).

\begin{tabular}{lccccccc}
\hline Data sets & $\begin{array}{c}\text { Horizontal } \\
\text { resolution }\end{array}$ & $\begin{array}{c}\text { Regions analyzed } \\
\text { (in pixels) }\end{array}$ & $\beta$ & $\alpha$ & $C_{1}$ & $H$ & References \\
\hline ETOPO5 (narrow strip) & $\approx 10 \mathrm{~km}$ & $500 \times 4000$ & 2.17 & 1.72 & 0.14 & 0.71 & - \\
ETOPO5 (full analysis) & $\approx 10 \mathrm{~km}$ & $2160 \times 4000$ & 2.12 & 1.81 & 0.13 & 0.69 & - \\
GTOPO30 & $\approx 1 \mathrm{~km}$ & $1225 \times 4096$ & 2.04 & 1.77 & 0.08 & 0.60 & - \\
U.S. & $90 \mathrm{~m}$ & $2500 \times 65536$ & 2.08 & 1.51 & 0.09 & 0.61 & - \\
Lower Saxony & $50 \mathrm{~cm}$ & $3000 \times 6000$ & 1.86 & 2.00 & 0.17 & 0.60 & - \\
Global parameters & - & - & - & 1.79 & 0.12 & - & See Sect. 6.2.5 \\
Deadman's Butte & $50 \mathrm{~m}$ & $512 \times 512$ & 1.93 & 1.9 & 0.045 & 0.51 & Lavallée et al. (1993) \\
French topography & $1 \mathrm{~km}$ & $512 \times 512$ & - & 1.7 & 0.075 & - & Lavallée et al. (1993) \\
U.S. & $90 \mathrm{~m}$ & $20 \times 512 \times 512$ & 1.91 & 1.70 & 0.07 & 0.52 & Pecknold et al. (1997) \\
\hline
\end{tabular}

data set. Because the spectrum of a scaling process is a decreasing power law, the amplitude of the $k_{\min }=1$ sinusoid is roughly proportional to $\Delta h_{\max }$ (or $E\left(k_{\min }\right) \Delta k \approx \Delta h_{\max }^{2}$ ) and the lowest possible amplitude (which is proportional to $\Delta h_{\min }$ ) roughly corresponds to the $k_{\max }$ sinusoid (or $\left.E\left(k_{\max }\right) \Delta k \approx \Delta h_{\min }^{2}\right)$, hence

$$
\left(\frac{\Delta h_{\max }}{\Delta h_{\min }}\right)^{2} \approx \frac{E\left(k_{\min }\right)}{E\left(k_{\max }\right)}=\left(\frac{k_{\min }}{k_{\max }}\right)^{-\beta}
$$

where $\beta$ is the spectral exponent. If the minimum/maximum heights on the transects $h_{\min }$ and $h_{\max }$ are measured in nondimensional digital counts and the wavenumbers in nondimensional inverse pixels, then we have

$k_{\max } \approx\left(h_{\max }-h_{\min }\right)^{2 / \beta}$

The wavenumbers at which this formula predicts that the spectra start to be corrupted are shown with arrows on Fig. 23. This approximate formula works particularly well for the U.S. spectrum, where it explains the drop in the high frequencies. In fact, this problem of insufficient dynamical range, which is related to the problem of insufficient statistics and the statistical nature of scale invariance (i.e. if more and more realizations are analyzed, the probability of having higher/lower altitudes increases, so it increases the dynamical range and the chance to observe good scaling over that range), can probably explain some scale breaks seen in the literature that are interpreted as characteristic scales of the process. There is also the problem of oversampling (see Sect. 4) that can lead to a fall-off of the spectra at high wavenumber

An important point is the fact that the spectra from our different data sets fall on a single straight line. Note here they are all in the same units and are only shifted in the vertical by a small multifractal correction determined by $\mathrm{K}(2)$ (see below). In addition, the range of scaling of each data set is roughly 3 orders of magnitude and their ranges overlap. This point is important: the overlap makes it clear that the high

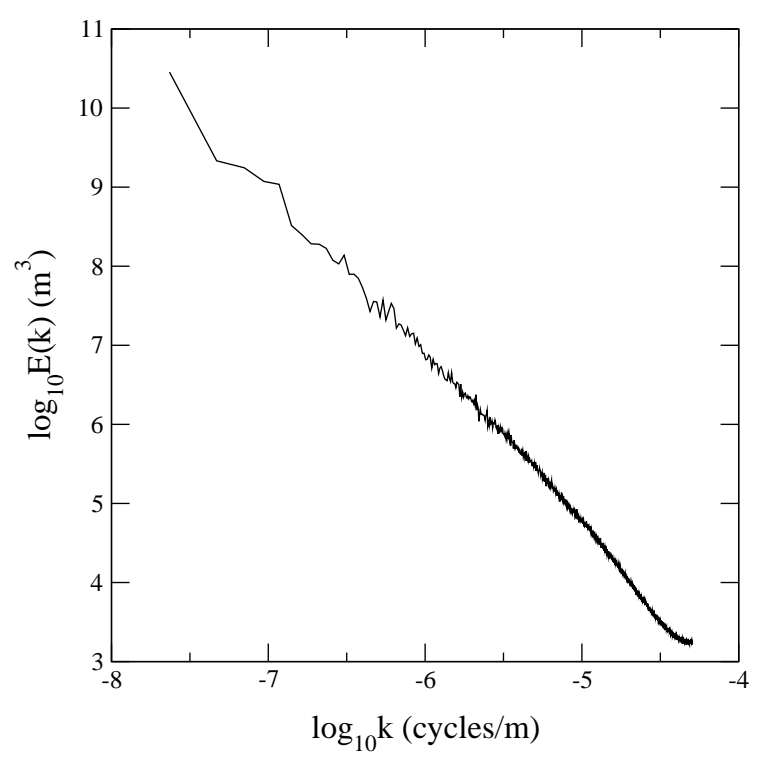

Fig. 24. $\log / \log$ plot of the spectral energy versus the wavenumber for ETOPO5 (full analysis).

wavenumber fall-offs or flattenings are artefacts of the particular data set. It also suggests that the corresponding scale breaks that are attributed to physical characteristic scales in the literature are artefacts. This result is quite different from that of Sayles and Thomas (1978) who argued that surfaces (natural and artificial) exhibited scaling over 8 orders of magnitude. In their case, the range of scaling of their individual data sets was very limited (they used 23 data sets to cover their 8 orders of magnitude) and the shifts in spectral amplitude were made more or less arbitrarily (Berry and Hannay, 1978 ) in order that they would line up over a wide range. In Fig. 23, only the Lower Saxony spectrum (with trees) does not follow the overall line, but the tree-free subsection does line-up. In our case, the vertical displacements are not arbitrary so the wide range of scaling is significant. 

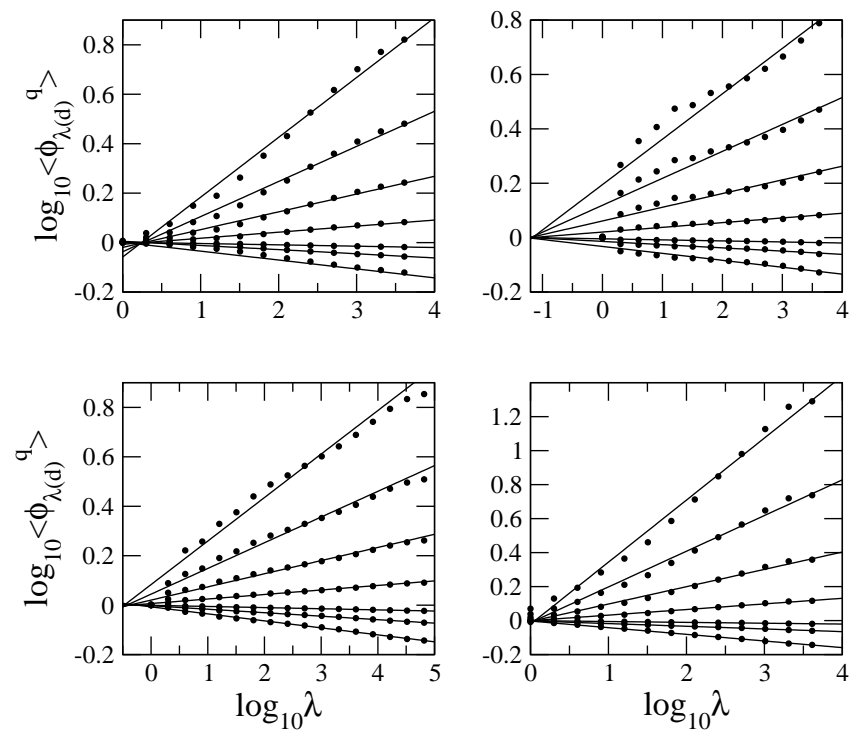

Fig. 25. $\mathrm{Log} / \log$ plot of the normalized trace moments versus the scale ratio $\lambda=L_{\mathrm{DEM}} / l$ for each DEMs. The values of $q$ of each trace moments are, from top to bottom, 2.18, 1.77, $1.44,1.17,0.04,0.12$ and 0.51 . Upper left corner: ETOPO5 (narrow strip analysis), $L_{\mathrm{ETOPO}}=40000 \mathrm{~km}$; upper right corner: GTOPO30, $L_{\mathrm{GTOPO} 30}=4096 \mathrm{~km}$; lower left corner: U.S. $\mathrm{DEM}, L_{\mathrm{US}}=5898 \mathrm{~km}$; lower right corner: Lower Saxony DEM, $L_{\text {Lower Saxony }}=3000 \mathrm{~m}$.

\subsubsection{Multifractal resolution corrections to spectral ampli- tudes}

The small shifts in the vertical are due to the resolution dependence of the multifractal noises $\phi_{\lambda}$ and the fact that the different data sets have quite different spatial resolutions. To see this, consider the fractional integrations implied by the convolutions in Table 1 . Since the Fourier transform of a convolution is a multiplication and the spectrum is determined by the square of the absolute transform, we have $E_{h}^{\mathrm{fBm}}(\mathbf{k})=E_{\phi}^{\mathrm{fBm}}(\mathbf{k})|\mathbf{k}|^{-(1+2 H)}$ and $E_{h}^{\mathrm{FIF}}(\mathbf{k})=E_{\phi}^{\mathrm{FIF}}(\mathbf{k}) \quad|\mathbf{k}|^{-2 H} \quad$ for the height spectra, where the noise spectra are $E_{\phi}^{\mathrm{fBm}}(\mathbf{k}) \propto \sigma^{2}$ and $E_{\phi}^{\mathrm{FIF}}(\mathbf{k}) \propto\left\langle\phi_{\lambda}^{2}\right\rangle|\mathbf{k}|^{-(1-K(2))}=\lambda^{K(2)}|\mathbf{k}|^{-(1-K(2))}$. We thus have

$$
\begin{aligned}
& E_{h}^{\mathrm{fBm}}(\mathbf{k}) \propto \sigma^{2}|\mathbf{k}|^{-(1+2 H)} \\
& E_{h}^{\mathrm{FIF}}(\mathbf{k}) \propto \lambda^{K(2)}|\mathbf{k}|^{-(1+2 H-K(2))}
\end{aligned}
$$

¿From Eq. (14) we can see that in addition to the multifractal correction to the spectral exponent, there is also a resolution dependent factor $\lambda^{K(2)}$, where $\lambda$ is the scale ratio which is inversely proportional to the resolution of the DEM. When comparing spectra from data sets having different intrinsic resolutions, there will be an additional offset of this amount. For example, using the nominal resolutions $10 \mathrm{~km}$,

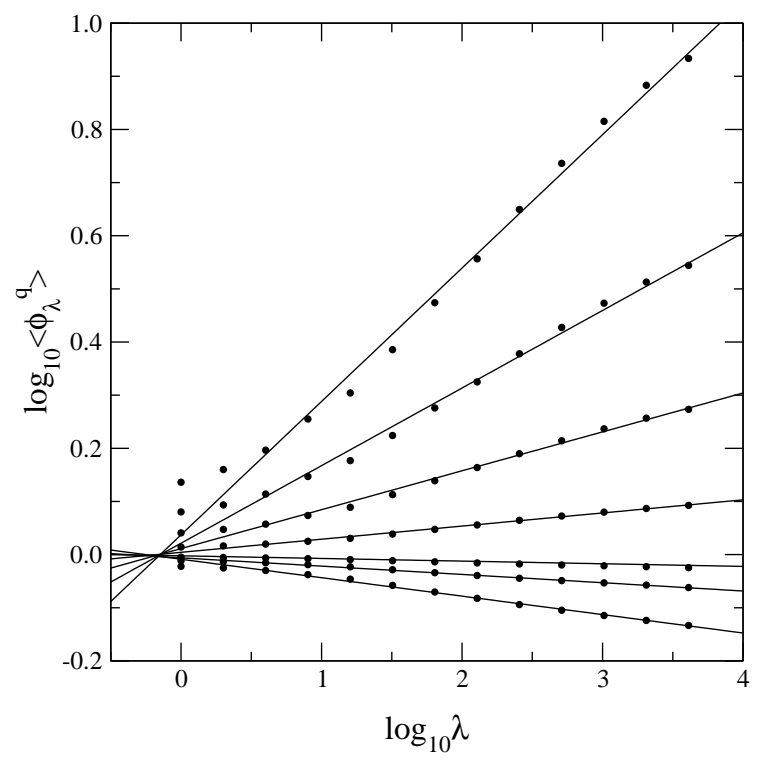

Fig. 26. Log/log plot of the normalized trace moments versus the scale ratio $\lambda=L_{\mathrm{ETOPO}} / l$ for ETOPO5 (full analysis). Here $L_{\text {ETOPO5 }}=40000 \mathrm{~km}$. The values of $q$ of each trace moments are, from top to bottom, 2.18, 1.77, 1.44, 1.17, 0.04, 0.12 and 0.51 .

$1 \mathrm{~km}, 90 \mathrm{~m}$ and $50 \mathrm{~cm}$ for the four different data sets, we see that using the "global" value $K(2)=0.22$ (see Sect. 6.2.5), the corrections necessary to bring them all to an "effective" $50 \mathrm{~cm}$ resolution are $8.84,5.32,3.13$ for $10 \mathrm{~km}, 1 \mathrm{~km}, 90 \mathrm{~m}$ respectively. We see from Fig. 23 that these corrections considerably improve the alignment of the spectra.

The spectra of Fig. 23 are from continents (except for ETOPO5, which is a continents/oceans mix). To calculate the theoretical spectral exponent predicted by the universal multifractal model, one must use the $H$ value of continents $\left(H_{\text {continents }}=0.66\right.$, see Table 4$)$ and the multifractal correction $K(2)$ (calculated with the global values of $\alpha$ and $C_{1}$ found with trace moments, see Sect. 6.2.5). This gives $\beta=1+2 H_{\text {continents }}-K(2)=2.10$. A reference line of slope -2.10 is shown on Fig. 23 to show that the overall slope is close to -2 (in rough agreement with Vening Meinesz (1951)), but is also close to the one predicted by universal multifractals (with parameters $\alpha=1.79, C_{1}=0.12$ and $H=0.66)$.

6.2.4 Trace moments analysis and the universal multifractal parameters

Trace moments analysis was performed on the four DEMs; see Figs. 25-26. Reasonable straight lines are obtained on all the DEMs, showing that Eq. (7) is obeyed. The slopes of the straight lines are equal to $K(q)$ : the fact that the slopes are nonzero implies that the $\mathrm{fBm}$ model is not adequate and their nonlinear variation with $q$ implies that the monofrac- 


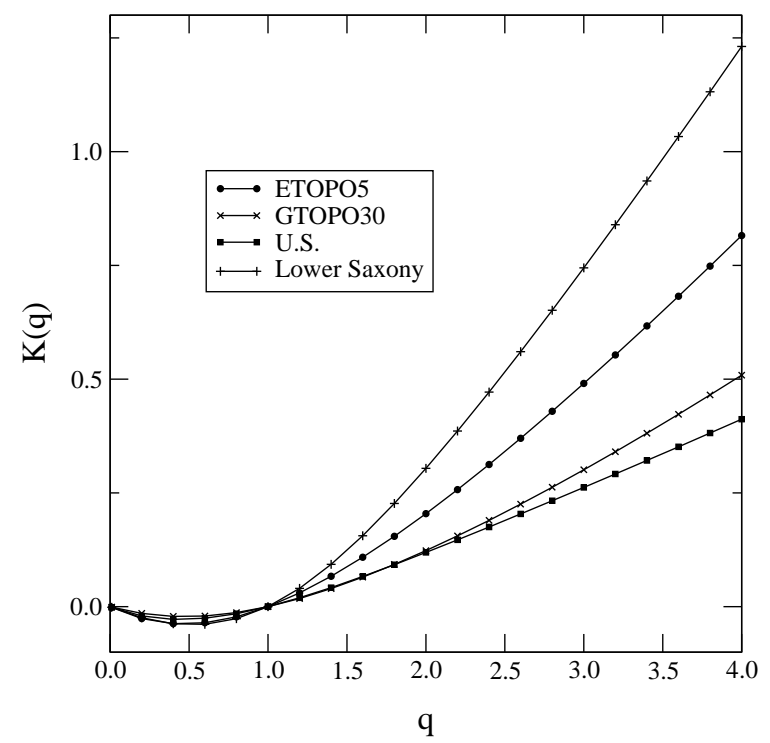

Fig. 27. Plot of the moment scaling function $K(q)$ as a function of the moment $q$ for the four DEMs (+'s correspond to Lower Saxony, squares to U.S., X's to GTOPO30 and circles to the narrow strip analysis of ETOPO5).

tal $\beta$-model is not adequate either (this is quantified below). The point at which all the trace moments converge corresponds to the "effective" outer scale of the cascade $L_{\text {eff. }}$ If there were an infinite ensemble of topographies, it would be the largest scale of the cascade. In the extreme case where we have a unique data set, $L_{\text {eff }}$ is bounded above by the scale of the data set; the trace moments will point to $\lambda=1$ (roughly as observed). If the scaling is broken, $\lambda=1$ would be less than the largest scale of the data set. It is interesting to note that the effective outer scale of ETOPO5 is $\approx 20000 \mathrm{~km}$ (see Fig. 25, upper left corner). This means that the variability of the topography is the same as that of a cascade starting at approximately $20000 \mathrm{~km}$, which is the size of a great circle (the maximum possible size on the earth). In the case of GTOPO30 (Fig. 25 upper right corner) and the U.S. DEM (Fig. 25 lower left corner), the effective outer scale is larger than the DEM outer scale, presumably differing slightly because the fitted lines on Fig. 25 (upper right and lower left corners) do not reproduce the flattening of the trace moments at high $\lambda$ (as discussed in Sect. 6.1.2).

To see more clearly the exact form of the corresponding $K(q)$ 's, these are shown on Figs. 27 and 28. Although the $K(q)$ 's are nonlinear, the asymptotes are linear. This linear behavior is the signature of a multifractal phase transition (Schertzer and Lovejoy, 1992). This phase transition is caused by the fact that, on a given realization, there is a maximum singularity $\gamma_{s}$ (the highest gradient on the dataset), implying that for $q>q_{s}, K(q)$ becomes linear:

$K(q)=\gamma_{s}\left(q-q_{s}\right)+K\left(q_{s}\right)$ for $q>q_{s}$

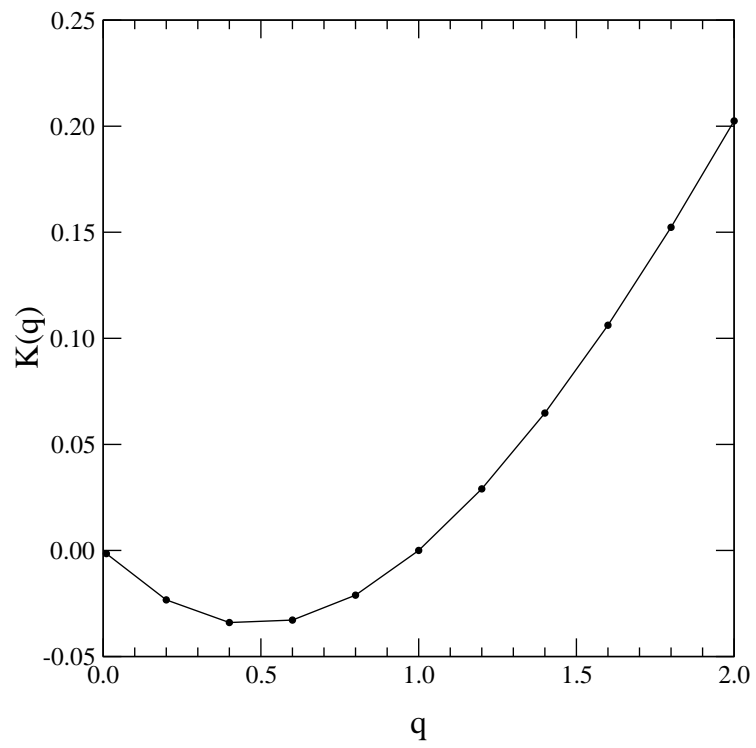

Fig. 28. Plot of the moment scaling function $K(q)$ as a function of the moment $q$ for ETOPO5 (full analysis).

From Eq. (15) and Fig. 27, we see that the values of $q_{s}$ can be estimated by measuring the value of $q$ where $K(q)$ becomes linear; we find $q_{s} \approx 3$ for all the data sets. For $q>q_{s}, K(q)$ is determined by the largest singularity present $\gamma_{s}$ (the statistics "saturate"). Note that these are second order multifractal phase transitions; the second derivative of $K(q)$ is discontinuous. First order transition due to divergence of statistical moments may also occur and may explain Bouchaud et al. (1993)'s results on fracture surfaces.

In order to test the universality hypothesis and estimate $\alpha$ and $C_{1}$, the $K(q)$ curves of Figs. 27 and 28 are fitted with Eq. (7) in the range $0<q<2<q_{s}$ (to obtain accurate moment estimates). The values obtained for the parameters can be seen in Table 5. These results can be compared to other studies on topography (see Table 5). Even if those analyses are on different parts of the world and are on different ranges of scales, the parameters obtained are not too different from those found here. Of course, it is important to remember that $\alpha$ and $C_{1}$ are statistical, and that the data sets here are larger. Note also that the parameters for the full analysis of ETOPO5 are quite similar to the narrow strip analysis; this justifies the use of only 500 transects to compare with other datasets.

\subsubsection{Global moment scaling function}

The similarity between the $\alpha$ and $C_{1}$ parameters between all these studies suggests that it may be possible to introduce a "global" $K(q)$ function that would describe the statistics of the Earth's "topographic" noise $\phi$ at all scales. This is certainly the simplest hypothesis that one could make about the Earth's noise. Even in the case with both continental 


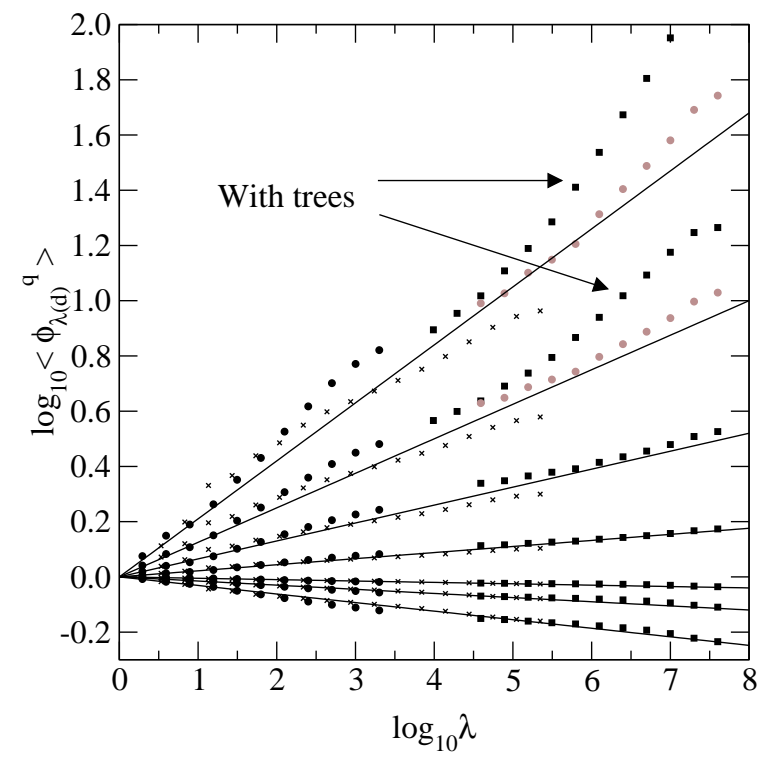

Fig. 29. $\log / \log$ plot of the normalized trace moments versus the scale ratio $\lambda=L_{\text {outer }} / l$ (with $L_{\text {outer }}=20000 \mathrm{~km}$ ) for the three DEMs (circles correspond to ETOPO5, X's to U.S. and squares to Lower Saxony). The solid lines are there to distinguish between each value of $q$ (from top to bottom, $q=2.18,1.77,1.44,1.17,0.04$, 0.12 and 0.51 ). The trace moments of the Lower Saxony DEM with trees for $q=1.77$ and $q=2.18$ are on the graph (indicated by arrows). The theoretical lines are computed with the global $K(q)$ function discussed in Sect. 6.2.5. Figure taken from Gagnon et al. (2003) with some modifications.

and oceanic data (ETOPO5), it is reasonable to make the hypothesis that they are described by the same $K(q)$, because the primary difference between the two appears to be $H$ (i.e. $\alpha$ and $C_{1}$ are similar, see Sect. 6.1.2). There are also morphological differences (i.e. anisotropies) between oceans and continents (and also between different places on oceans/continents), but they are largely washed out by the use of isotropic statistics (angle integrated power spectra, coarse graining over squares at all scales in trace moments).

So here we argue that for $\phi$, the statistics are the same at all scales and can be described by a global $K(q)$ function. A simple way to obtain an estimate of the global $K(q)$ is to take the average of the parameters obtained in this study $\left(\alpha=1.79 \pm 0.18\right.$ and $C_{1}=0.12 \pm 0.04$, where the uncertainties are equal to one standard deviation). To see whether this global $K(q)$ is plausible we must test that the cascade is continuous from planetary scales down to small scales, i.e. to check whether the trace moments of all the data sets lie on straight lines when put on a single graph. However, we cannot mechanically superpose the trace moments for the different regional data sets. This is because (recall from Sect. 5.2) each regional data set will satisfy $\log \left\langle\phi_{\lambda}^{q}\right\rangle=1$ at $\lambda=1$ (with $\lambda=1$ corresponding to the outer scale of the regional data, i.e. different for each). The reason for this apparent contra- diction is that the regional data sets are missing variability due to the planetary scales down to their outer scales. However, knowing the global $K(q)$, we can estimate this missing variability and adjust the trace moments by a theoretically determined amount. The first step is to fix the outer scale $L_{\text {outer }}$ of the entire process, approximately $20000 \mathrm{~km}$ (see Fig. 25). Next we calculate $\lambda=L_{\text {outer }} / l$ of each DEM (this corresponds to a shift of the trace moments on the horizontal axis). Then, in order to take into account the fact that the variability of the regional data sets is artificially low, we use the basic factorization property of cascades. Let $\lambda_{\text {large }}=L /$ (data set scale) be the ratio of the missing range of scales. If $\lambda_{\text {small }}=($ data set scale $) /($ pixel scale), then factorization (valid for the cascade quantity $\phi$ but not directly for $h$ ) means

$\left\langle\phi_{\lambda_{\text {large }} \lambda_{\text {small }}}^{q}\right\rangle=\left\langle\phi_{\lambda_{\text {large }}}^{q}\right\rangle\left\langle\phi_{\lambda_{\text {small }}}^{q}\right\rangle=\lambda_{\text {large }}^{K(q)}\left\langle\phi_{\lambda_{\text {small }}}^{q}\right\rangle$

where $K(q)$ is the globally averaged $K(q)$ discussed previously and $\left\langle\phi_{\lambda_{\text {small }}}^{q}\right\rangle$ is obtained directly from the analysis of each data set. On a $\log / \log$ plot, the multiplicative factor due to the variability of the larger scales becomes a linear shift in the trace moments, corresponding to a theoretically predicted linear vertical shift in Fig. 25. Figure 29 shows the trace moments of three DEMs shifted according to Eq. (16) (the trace moments of GTOPO30 are not shown in Fig. 29 because its scaling range is already contained in the U.S. range, so we avoid overcrowding the graph).

As can be seen from Fig. 29, the lines obtained are not exactly straight, but oscillate slightly around a straight line (this is more apparent for large values of $q$ ). These oscillations may be due to scaling anisotropies, which are only partially eliminated by our isotropic analyses (see the ensemble spectrum of "Mt. Multi" in Sect. 2.3 for a similar type of behaviour due to anisotropy). With this caveat, the trace moments obtained on Fig. 29 are nearly straight and continuous over nearly 6 orders of magnitude (there is a break at $\approx 40 \mathrm{~m}$ that is probably due to trees (Gagnon, 2001)), meaning that the Earth's topography is multiscaling over that range. We can quantify this statement on multifractality by estimating the error of the fit. To do this, we calculate the deviation (normalized residuals) $\Delta$ between theory (i.e. the straight lines on Fig. 29) and data with the following formula:

$\Delta=\left|\log _{10}\left(\left\langle\phi_{\lambda}^{q}\right\rangle\right)^{1 / q}-\log _{10}\left(\lambda^{K(q)}\right)^{1 / q}\right|$

where $K(q)$ is the global moment scaling function discussed above and we average over $0<q<2$ and the scale range $20000 \mathrm{~km}$ to $40 \mathrm{~m}$. For the estimated global parameters $\alpha=1.79$ and $C_{1}=0.12$, we get $\bar{\Delta}=0.16$, corresponding to $\pm 10^{\bar{\Delta}}= \pm 45 \%$ in the statistical moments, with the overbar indicating averaging over all $\lambda$ 's and $q$ 's. Considering that we are dealing with four different data sets that cover nearly 6 orders of magnitude in scale, that we are estimating all $q<2$ moments and that the fit is done with only two parameters 
(with no optimization of the parameters nor minimization of error), then the fit is fairly good. This is an indication that the hypothesis of a global $K(q)$ is a reasonable assumption for the isotropic noise. In contrast, the use of a multiplicative monofractal model $(\alpha=0)$ to fit all the curves would give $\bar{\Delta}=0.55$ (or $\pm 355 \%$ in the statistical moments). Fractional Brownian motion cannot be used to explain these results either, because $K(q) \neq 0$ whereas $\mathrm{fBm}$ predicts $K(q)=0$. For example, taking a typical well estimated trace moment at $q=2.17$, we obtain a slope $K(2.17)=0.24 \pm 0.03$, which is 8 standard deviations away from zero. For other $q$ and other data sets, $K(q)$ is typically $5-10$ standard deviations from zero. The trace moments are therefore not flat, definitely ruling out the monofractal $\mathrm{fBm}$ model.

\section{Conclusions}

The earth's topography is a fundamental geosurface. It is important in numerous applications and its immediacy has challenged generations of mathematicians, physicists and geoscientists to devise realistic descriptions and models. Its high variability over huge ranges of scales continues to defy traditional scale bound notions of variability. In this paper we showed how to tackle this variability using scaling ideas. We first showed how new ideas of scaling can be used to make realistic models of topography with interesting morphologies. We then showed that in real topography scaling is accurately obeyed over wide ranges of scale and quantified that statement.

Surprisingly, the notions of scaling used in modeling and analyzing the earth's surface have not changed much since their early development: they are still generally quasiGaussian (the monofractal $\mathrm{fBm}$ ). To appreciate the restrictive nature of this framework, we recalled that scaling models are generally based on mathematical singularities which are the basic scaling functions. A general feature of such singular models is that they produce large, strong structures which can stand out even in the limit when an infinite number are superposed. The Gaussian case is exceptional in this regard; the morphology of the singularities gets "washed out" in the infinite limit since all singularities have nearly the same strengths. In order to achieve interesting and realistic morphologies/textures, we need not only more wild statistical variability but also singularities with interesting shapes. While the former is possible using multifractals, the latter is possible with the use of scale changing operators, generators and scale functions, i.e. in the framework of Generalized Scale Invariance. We illustrate the resulting subtle interplay between singularity shapes, statistical variability and resulting morphology with many examples from numerical simulations. From the simulations, one can see that multifractals give rise to subtle hierarchies of mountains and plains that are very appealing visually when compared to $\mathrm{fBm}$ or fLm. We also show with the help of simulations and pedagogical examples, that the hypothesis of wide range scaling is well defined and not in contradiction with other results in the literature. The existence of apparent breaks in the scaling is in fact a result of normal multifractal variability.

All of this shows that anisotropic multifractals hold promise for modelling topography. But this is only a proof of principle: what about real topography? In the second part of the paper, we showed that, indeed, topography is multiscaling over the largest scale range - nearly 6 orders of magnitude - and on the largest data sets - a hundred times the data used in previous studies. Restricting ourselves to isotropic statistics (i.e. averaged over all directions), the fundamental result is that from planetary scales down to roughly $40 \mathrm{~m}$ (broken apparently due to the indirect effect of trees), the scaling is remarkably accurately followed. Indeed, not only can the noise statistics for weak and strong gradients (low and high order moments) be well represented by a unique moment scaling function $K(q)$ parametrized by two basic exponents $\left(\alpha\right.$ and $C_{1}$ ), the overall agreement between the noise statistics and that predicted by the two parameters is $\pm 45 \%$. In contrast, the $\beta$-model (monofractal noise, $\alpha=0$ ) gives an error of $\pm 355 \%$ and the $\mathrm{fBm}$ (homogeneous noise, $K(q)=0)$ is typically $5-10$ standard deviations away from the data, hence the inadequacy of monofractal models.

By systematically comparing various subregions of the globe, we also showed that while the continents, oceans and continental margins have roughly the same noise statistics (charaterized by $\alpha$ and $C_{1}$ ), their height statistics (characterized by $H$ ) were significantly different (see Table 4 ). This suggests that the physical mechanisms responsible for $H$ (whatever they are) are different, while the ones responsible for $\alpha$ and $C_{1}$ are similar. It also justifies the use of a unique global moment scaling to characterize the Earth's topography as a whole (see Table 5 for a summary of the parameters obtained).

While the finding that each topographic level set has its own fractal dimension surely restores a degree of realism to the popular monofractal picture of topography, the analyses done in this paper are isotropic (angle averaged): the scale by scale and location by location anisotropy has largely been washed out. As discussed previously, the development of Generalized Scale Invariance has shown that - at least in principle - realism can be restored by allowing the notion of scale itself to be defined by the physics. In other words, Generalized Scale Invariance could be used to model topographies with various morphologies in different locations - where the scale changing operator $T_{\lambda}$ would be constrained by the physics responsible for these morphologies - all of this in a scaling framework. The development of realistic topographic models and the corresponding anisotropic analysis techniques are clearly the subjects of future research.

The "complete explanation" of topography can then be roughly divided in two parts: its description and its underlying physics, with a non-trivial link between the two. This paper mostly deals with the description part and an implied 
symmetry constraint on the underlying mechanisms. An accurate and complete characterization of the statistical properties of topography is important in applications where it is used as a boundary, such as atmospheric/oceanic currents models, synthetic aperture radar interferograms or radar scattering models. It potentially explains many of the classical scaling relations of river basin hydrology. More generally, just as energy conservation is an often used model constraint, so should scale invariance: scale invariance and multifractality can be useful model selection criteria.

Acknowledgements. The authors would like to thank A. Carsteanu and two other anonymus NPG reviewers for their constructive comments. We also thank anonymous reviewers from JGR Solid Earth, JGR Earth Surface and Geophys. J. Internat. We also thank the German Aerospace Center (DLR)'s Institute of Space Sensor Technology and Planetary Exploration for providing the high resolution DEM of Lower Saxony. This work was supported in part by the GEOTOP at Université du Québec à Montréal.

Edited by: B. D. Malamud

Reviewed by: A. A. Cârsteanu and two other referees

\section{References}

Alvarez, A., Hernandez-Garcia, E., and J. Tintoré: On the effect of small-scale oceanic variability on topography-generated currents, Geophys. Res. Lett., 27(6), 739-742, 2000.

Audit, B., Bacry, E., Muzy, J. F., and Arnéodo, A.: Wavelet-based estimators of scaling behavior, IEEE Transactions on Information Theory, 48(11), 2938-2954, 2002.

Aviles, C. A., Scholz, C. H., and Boatwright, J.: Fractal analysis applied to characteristic segments of the San Andreas fault, J. Geophys. Res., 92(B1), 331-344, 1987.

Balmino, G., Lambeck, K., and Kaula, W.: A spherical harmonic analysis of the Earth's topography, J. Geophys. Res., 78(2), 478481, 1973.

Balmino, G.: The spectra of the topography of the Earth, Venus and Mars, Geophys. Res. Lett., 20(11), 1063-1066, 1993.

Banavar, J. R., Colaiori, F., Flammini, A., Maritan, A., and Rinaldo, A.: Scaling, optimality, and landscape evolution, J. Stat. Phys., 104(112), 1-48, 2001.

Barenblatt, G. I., Zhivago, A. V., Neprochnov, Y. P., and Ostrovskiy, A. A.: The fractal dimension: a quantitative characteristic of ocean-bottom relief, Oceanology, 24(6), 695-697, 1984.

Bell, T. H.: Statistical features of sea-floor topography, Deep-Sea Res., 22, 883-892, 1975.

Berkson, J. M. and Matthews, J. E.: Statistical properties of seafloor roughness, in: Acoustics and the Sea-Bed, edited by: Pace, N. G., Bath University Press, Bath, England, 215-223, 1983.

Berry, M. V. and Hannay, J. H.: Topography of random surfaces, Nature, 273, 573, 1978.

Bouchaud, E., Lapasset, G., Planès, J., and Naveos, S.: Statistics of branched fracture surfaces, Phys. Rev. B, 48, 2917-2928, 1993.

Burrough, P. A.: Fractal dimensions of landscapes and other environmental data, Nature, 294, 240-242, 1981.

Chase, C. G.: Fluvial landsculpting and the fractal dimension of topography, Geomorphology, 5, 39-57, 1992.
Data Announcement 88-MGG-02: Digital relief of the Surface of the Earth, NOAA, National Geophysical Data Center, Boulder, Colorado, 1988.

de Wisj, H. J.: Statistics of ore distributions Part I: Frequency distributions of assay values, Geol. Mijnb, 13, 127-144, 1951.

Dodds, P. S. and Rothman, D. H.: Scaling, universality, and geomorphology, Annu. Rev. Earth Planet. Sci., 28, 571-610, 2000.

Dietler, G. and Zhang, Y.: Fractal aspects of the Swiss landscape, Physica A, 191, 213-219, 1992.

Fox, C. G. and Hayes, D. E.: Quantitative methods for analyzing the roughness of the seafloor, Rev. Geophys., 23, 1-48, 1985.

Gagnon, J.-S.: Multiscaling and multifractality in the Earth's topography, M.Sc., McGill University, Montréal, Canada, 2001.

Gagnon, J.-S., Lovejoy, S., and Schertzer, D.: Multifractal surfaces and terrestrial topography, Europhys. Lett., 62(6), 801807, 2003.

Gallant, J. C., Moore, I. D., Hutchinson, M. F., and Gessler, P.: Estimating fractal dimension of profiles: a comparison of methods, Mathematical Geology, 26(4), 455-481, 1994.

Gaonac'h, H., Lovejoy, S., and Schertzer, D.: Multifractal analysis of infrared imagery of active thermal features at Kilauea volcano, Int. J. Rem. Sens., 24(11), 2323-2344, 2003.

Gibert, D. and Courtillot, V.: Seasat altimetry and the South Atlantic geoid 1. Spectral analysis, J. Geophys. Res., 92(B7), 62356248, 1987.

Gilbert, L. E.: Are topographic data sets fractal?, PAGEOPH, 131, 241-254, 1989.

Goodchild, M. F.: Fractals and the accuracy of geographical measures, Mathematical Geology, 12(2), 85-98, 1980.

Guarnieri, A. M.: SAR interferometry and statistical topography, IEEE Transact, Geosci. Rem. Sens., 40(12), 2567-2581, 2002.

Harvey, D. C., Gaonac'h, H., Lovejoy, S., Stix, J., and Schertzer, D.: Multifractal characterization of remotely sensed volcanic features: A case study from the Kilauea volcano, Hawaii, Fractals, 10, 265-274, 2002.

Heiskanen, W. A. and Vening Meinesz, F. A.: The Earth and its gravity field, McGraw-Hill, New-York, 1958.

Herzfeld, U. C., Kim, I. I., and Orcutt, J. A.: Is the ocean floor a fractal?, Mathematical Geology, 27(3), 421-462, 1995.

Herzfeld, U. C. and Overbeck, C.: Analysis and simulation of scaledependent fractal surfaces with application to seafloor morphology, Computers \& Geosciences, 25, 979-1007, 1999.

Huang, J. and Turcotte, D. L.: Fractal mapping of digitized images: application to the topography of Arizona and comparisons with synthetic images, J. Geophys. Res., 94(B6), 7491-7495, 1989.

Huang, J. and Turcotte, D. L.: Fractal image analysis: application to the topography of Oregon and synthetic images, J. Opt. Soc. Am. A, 7(6), 1124-1130, 1990.

Hurst, H. E.: Long-term storage capacity of reservoirs, Transactions of the American Society of Civil Engineers, 116, 770-808, 1951.

Kardar, M., Parisi, G., and Zhang, Y.-C.: Dynamic scaling of growing interfaces, Phys. Rev. Lett., 56, 889-892, 1986.

Klinkenberg, B. and Goodchild, M. F.: The fractal properties of topography: a comparison of methods, Earth Surface Processes and Landforms, 17, 217-234, 1992.

Lambeck, K.: Geophysical geodesy: the slow deformations of the Earth, Oxford University Press, New York, 1988.

Land Processes Distributed Active Archive Center: GTOPO30, EROS data center, Sioux Falls, South Dakota, 1996. 
Lavallée, D., Lovejoy, S., Schertzer, D., and Ladoy, P.: Nonlinear variability of landscape topography: multifractal analysis and simulation, in: Fractals in Geography, edited by: De Cola, L. and Lam, N., Prentice-Hall, New Jersey, 158-192, 1993.

Leary, P.: Rock as a critical-point system and the inherent implausibility of reliable earthquake prediction, Geophys. J. Internat., 131, 451-466, 1997.

Lewis, G. M., Pecknold, S., Lovejoy, S., and Schertzer, D.: The scale invariant generator technique for quantifying anisotropic scale invariance, Computers and Geosciences, 25(9), 963-978, 1999.

Lovejoy, S. and Schertzer, D.: Our multifractal atmosphere: A unique laboratory for non-linear dynamics, Physics in Canada, 46(4), 62-71, 1990.

Lovejoy, S. and Schertzer, D.: Multifractal analysis techniques and the rain and cloud fields from $10^{-3}$ to $10^{6} \mathrm{~m}$, in: Non-linear Variability in Geophysics, edited by: Schertzer, D. and Lovejoy, S., Kluwer Academic Publishers, Netherlands, 111-144, 1991.

Lovejoy, S., Lavallée, D., Schertzer, D., and Ladoy, P.: The $l^{1 / 2}$ law and multifractal topography: theory and analysis, Nonlin. Processes Geophys., 2, 16-22, 1995,

http://www.nonlin-processes-geophys.net/2/16/1995/.

Lovejoy, S., Pecknold, S., and Schertzer, D.: Stratified multifractal magnetization and surface geomagnetic fields, part 1: spectral analysis and modelling, Geophys. J. Inter., 145, 112-126, $2001 \mathrm{a}$.

Lovejoy, S., Schertzer, D., Tessier, Y., and Gaonac'h, H.: Multifractals and resolution-independent remote sensing algorithms: the example of ocean color, Int. J. Remote Sensing, 22, 1191-1234, $2001 \mathrm{~b}$.

Lovejoy, S., Schertzer, D., and Gagnon, J.-S.: Multifractal simulations of the Earth's surface and interior: anisotropic singularities and morphology, in: GIS and Spatial Analysis, Proc. of the Inter. Assoc. Math. Geology 2005, edited by: Cheng, Q. and BonhamCarter, G., 37-54, 2005.

Mandelbrot, B. B.: How long is the Coast of Britain?, Statistical self-similarity and fractional dimension, Science, 156, 636-638, 1967.

Mandelbrot, B. B.: Stochastic models for the Earth's relief, the shape and the fractal dimension of the coastlines, and the number-area rule for islands, Proc. Nat. Acad. Sci. USA, 72(10), 3825-3828, 1975.

Mandelbrot, B. B.: The Fractal Geometry of Nature, Freeman, New York, 1983.

Mandelbrot, B. B. and van Ness, J. W.: Fractional Brownian motions, fractional noises and applications, SIAM review, 10, 422 450, 1968.

Mareschal, J.-C.: Fractal reconstruction of sea-floor topography, PAGEOPH, 131, 197-210, 1989.

Mark, D. M. and Aronson, P. B.: Scale-dependent fractal dimensions of topographic surfaces: an empirical investigation, with applications in geomorphology and computer mapping, Mathematical Geology, 16(7), 671-683, 1984.

Marsan, D. and Bean, C. J.: Multiscaling nature of sonic velocities and lithography in the upper crystalline crust: evidence from the KTB main borehole, Geophys. Res. Lett., 26, 275-278, 1999.

Morel, S., Schmittbuhl, J., Bouchaud, E., and Valentin, G.: Scaling of crack surfaces and implications for fracture mechanics, Phys. Rev. Lett., 85, 1678-1681, 2000.

Muzy, J. F., Bacry, E., and Arnéodo, A.: Multifractal formal- ism for fractal signals: The structure-function approach versus the wavelet-transform modulus-maxima method, Phys. Rev. E, 47(2), 875-884, 1993.

Okubo, P. G. and Aki, K.: Fractal geometry in the San Andreas fault system, J. Geophys. Res., 92(B1), 345-355, 1987.

Orosei, R., Bianchi, R., Coradini, A., Espinasse, S., Federico, C., Ferriccioni, A., and Gavrishin, A. I.: Self-affine behaviour of Martian topography at kilometre scale from Mars Orbiter Laser Altimeter data, J. Geophys. Res., 108(E4), 8023-8032, 2003.

Pandey, G., Lovejoy, S., and Schertzer, D.: Multifractal analysis of daily river flows including extremes for basins of five to two million square kilometers, one day to 75 years, J. Hydrol., 208, 62-81, 1998.

Parsons, B. and Sclater, J. G.: An analysis of the variation of ocean floor bathymetry and heat flow with age, J. Geophys. Res., 82(5), 803-827, 1977.

Pecknold, S., Lovejoy, S., and Schertzer, D.: Stratified multifractal magnetization and surface geomagnetic fields, part 2: multifractal analysis and simulation, Geophys. J. Inter., 145, 127-144, 2001.

Pecknold, S., Lovejoy, S., Schertzer, D., and Hooge, C.: Multifractal and resolution dependence of remotely sensed data: GSI and GIS, in: Scale in Remote Sensing and GIS, edited by: Quattrochi, D. A. and Goodchild, M. F., CRC Press, 361-394, 1997.

Perrin, J.: Les atomes, NRF-Gallimard, Paris, 1913.

Pilkington, M. and Todoeschuck, J.: Scaling nature of crustal susceptibilities, Geophys. Res. Lett., 22, 779-782, 1995.

Prey, A.: Darstellung der Höhen- und Tiefenverhältnisse der Erde durch eine Entwicklung nach Kugelfunktionen bis zur 16. Ordnung, in: Abhandl. Ges. Wiss. Göttingen, Math.-physik. Kl., 11(1), 1-29, 1922.

Richardson, L. F.: The problem of contiguity: an appendix to statistics of deadly quarrels, Gen. Syst. Yearb., 6, 139-187, 1961.

Rinaldo, A., Maritan, A., Colaiori, F., Flammini, A., Rigon, R., Rodriguez-Iturbe, I., and Banavar, J. R.: Thermodynamics of fractal networks, Phys. Rev. Lett., 76, 3364-3368, 1996.

Rodriguez-Iturbe, I. and Rinaldo, A.: Fractal River Basins: Chance and Self-Organization, Cambridge University Press, Cambridge, 1997.

Rinaldo, A., Rodriguez-Iturbe, I., Rigon, R., Bras, R. L., IjjaszVasquez, E., and Marani, A.: Minimum energy and fractal structures of drainage networks, Water Resour., 28(9), 2183-2195, 1992.

Rinaldo, A., Rodriguez-Iturbe, I., Rigon, R., Ijjasz-Vasquez, E., and Bras, R. L.: Self-organized fractal river networks, Phys. Rev. Lett., 70, 822-825, 1993.

Sayles, R. S. and Thomas, T. R.: Surface topography as a nonstationary random process, Nature, 271, 431-434, 1978.

Schertzer, D. and Lovejoy, S.: Generalized scale invariance in turbulent phenomena, Physico-Chemical Hydrodynamics Journal, 6, 623-635, 1985.

Schertzer, D. and Lovejoy, S.: Physical modeling and analysis of rain and clouds by anisotropic scaling multiplicative processes, J. Geophys. Res., 92(D8), 9693-9714, 1987.

Schertzer, D. and Lovejoy, S.: Nonlinear geodynamical variability: multiple singularities, universality and observables, in: Nonlinear Variability in Geophysics, edited by: Schertzer, D. and Lovejoy, S., Kluwer Academic Publishers, Netherlands, 41-82, 1991. 
Schertzer, D. and Lovejoy, S.: Hard and soft multifractal processes, Physica A, 185, 187-194, 1992.

Schertzer, D. and Lovejoy, S.: From scalar cascades to Lie cascades: Joint multifractal analysis of rain and cloud processes, in: Space/time variability and interdependence for various hydrological processes, edited by: Feddes, R. A., Cambridge University Press, 153-173, 1995.

Schertzer, D. and Lovejoy, S.: Universal multifractals do exist!, Journal of Applied Meteorology, 36, 1296-1303, 1997.

Schmittbuhl, J., Schmitt, F., and Scholz, C.: Scaling invariance in crack surfaces, J. Geophys. Res., 100(B4), 5953-5973, 1995.

Sclater, J. G., Anderson, R. N., and Lee Bell, M.: Elevation of ridges and evolution of the Central Eastern Pacific, J. Geophys. Res., 76(32), 7888-7915, 1971.

Sinclair, K., and Ball, R.: A mechanism for global optimization of river networks from local erosion rules, Phys. Rev. Lett., 76, 3360-3363, 1996.

Sornette, D. and Zhang, Y.-C.: Non-linear Langevin model of geomorphic erosion processes, Geophys. J. Int., 113, 382-386, 1993.

Steinhaus, H.: Length, shape and area, Colloquium Mathematicum, III, 1-13, 1954.

Tchiguirinskaia, I., Lu, S., Molz, F. J., Williams, T. M., and Lavallée, D.: Multifractal versus monofractal analysis of wetland topography, Stochastic Environmental Research and Risk Assessment, 14, 8-32, 2000.

Tessier, Y., Lovejoy, S., Hubert, P., and Schertzer, D.: Multifractal analysis and modeling of rainfall and river flows and scaling, causal transfer functions, J. Geophys. Res., 101, 427-440, 1996.

Turcotte, D. L.: Fractals in geology and geophysics, PAGEOPH, 131, 171-196, 1989.
Turcotte, D. L.: Fractals and chaos in geology and geophysics, Cambridge University Press, Cambridge, 1992.

Turcotte, D. L. and Oxburgh, E. R.: Finite amplitude convective cells and continental drift, J. Fluid Mech., 28(part 1), 29-42, 1967.

United States Geological Survey: Digital Elevation Models, National Mapping Program Technical Instructions, Data Users Guide, U.S. Geological Survey, Second printing (revised), Reston, Virginia, 1990.

Veneziano, D. and Iacobellis, V.: Self-similarity and multifractality of topographic surfaces at basin and subbasin scales, J. Geophys. Res., 104(B6), 12 797-12 812, 1999.

Veneziano, D. and Niemann, J. D.: Self-similarity and multifractality of fluvial erosion topography 1 . Mathematical conditions and physical origin, Water Resour. Res., 36(7), 1923-1936, 2000.

Veneziano, D. and Niemann, J. D.: Self-similarity and multifractality of fluvial erosion topography 2 . Scaling properties, Water Resour. Res., 36(7), 1937-1951, 2000.

Vening Meinesz, F. A.: A remarkable feature of the Earth's topography, origin of continents and oceans, Proc. Koninkl. Ned. Akad. Wetensch. ser. B, 55, 212-228, 1951.

Weissel, J. K., Lincoln, F. P., and Malinverno, A.: The lengthscaling properties of topography, J. Geophys. Res., 99(B7), 13 997-14 012, 1994.

Wewel, F., Scholten, F., and Gwinner, K.: High resolution stereo camera (HRSC) - Multispectral 3D data acquisition and photogrammetric data processing, Canadian Journal of Remote Sensing, 26(5), 466-474, 2000.

$\mathrm{Xu}$, T., Moore, I. D., and Gallant, J. C.: Fractals, fractal dimensions and landscapes - a review, Geomorphology, 8, 245-262, 1993. 